\title{
El poder transformador de los saberes en paisajes de terrazas
}

\author{
The Transformative Power of Knowledge in Terraced Landscapes
}

\author{
María Angélica Salas* \\ Universidad de Coventry \\ https://orcid.org/0000-0003-4295-397X \\ mas49@gmx.ney \\ Timmi Tillmann \\ Universidad de Coventry \\ https:// orcid.org/0000-0001-5325-2293 \\ TimmiTillmann@gmail.com
}

Recibido: 29/07/2020; Revisado: 18/11/2020; Aceptado: 20/11/2020

\begin{abstract}
Resumen
La agricultura transforma la naturaleza y forja los saberes bioculturales de las sociedades que viven y producen en paisajes de terrazas. Argumentamos conceptualmente la vitalidad epistemológica de estos saberes en la sociedad global presentándolos en una selección de percepciones locales resultantes de la aplicación de herramientas metodológicas o técnicas propias de la Investigación Acción Participativa aplicada en zonas de montaña en Asia, Europa y América Latina. Finalizamos reflexionando sobre los saberes bioculturales y la sostenibilidad en la sociedad global con miras a la justicia cognitiva.
\end{abstract}

Palabras clave: terrazas agrícolas, saberes bioculturales, Investigación Acción Participativa, justicia cognitiva.

\begin{abstract}
This article deals with agriculture as a transformative human action that shapes the knowledge of groups living and producing in terraced landscapes. We present the perceptions, concepts, values and visions of these groups within the context of biocultural diversity. This analysis is based on the methodological application of Participatory Action Research in mountainous communities in Asia, Europe and Latin America. Finally, we reflect on the destiny of biocultural diversity and sustainability, indicating that empowerment of local initiatives will yield democratic spaces for the coexistence of local wisdoms aiming for cognitive justice.
\end{abstract}

Key words: Agricultural Terraces, Biocultural Wisdom, Participatory Action Research, Cognitive Justice.

*Autor de correspondencia / Corresponding author.

Copyright: (C) 2021 ULPGC. Este es un artículo de acceso abierto distribuido bajo los términos de la licencia Creative Commons Atribución-NoComercial-SinDerivar (by-nc-nd) Spain 3.0. 


\section{INTRODUCCIÓN}

Inscribimos el tema de los saberes transformadores dentro del marco de la historia de la agricultura y de los sistemas de conocimiento de los pueblos que mantienen una especial relación con la naturaleza para producir alimentos. Planteamos que esta relación cobra especial relevancia constatando que en la actualidad los saberes que sustentan el mosaico de sistemas de agricultura de pequeña escala, incluyendo a las terrazas, alimentan a la gran mayoría de la humanidad.

Nos basamos en antecedentes conceptuales que visibilizan las propiedades epistemológicas de los saberes con una mirada antropológica saliendo de los esquemas dicotómicos que consideran que los conocimientos científicos modernos son superiores y los tradicionales inferiores. Nos inscribimos en los estudios interdisciplinarios que ponen énfasis en el concepto de la diversidad biocultural como clave de la sostenibilidad y en el reconocimiento democrático que los saberes plurales, los estilos de vivir y de producir en diversidad tienen derecho a coexistir pacíficamente en este mundo global.

\section{OBJETIVOS Y METODOLOGÍA}

Este artículo tiene la intención de invitar a una reflexión teórica y metodológica sobre la presencia y relevancia de los saberes locales con relación a la sostenibilidad de los paisajes de terrazas. Para ello pasamos revista a la historia de la agricultura y nos valemos de la perspectiva de los saberes de diversidad biocultural como horizonte conceptual. Argumentamos que los paisajes de terrazas forman parte de la producción de pequeña escala que alimenta a la mayor parte del mundo y por ello, las percepciones, los conceptos, ideas, valores de los grupos merecen el reconocimiento social con justicia cognitiva a nivel global.

Los saberes que forman parte sustancial del contenido de este artículo surgieron de la aplicación de la Investigación Acción Participativa (IAP) entre 2010 y 2018. Específicamente se llevó a cabo en el contexto de la realización de los cuatro Congresos Mundiales de Terrazas, organizados en cada caso por instituciones y organizaciones locales en colaboración con ITLA (Alianza Internacional de Paisajes con Terrazas). Nos parece relevante precisar que la metodología IAP, se empleó dentro del marco general de estos congresos en distintas modalidades de procesos grupales abiertos como seminarios, talleres rurales, trabajos y visitas de campo (SAlAS, 2010). Estos sitúan las experiencias de mujeres y hombres al centro del diálogo en el que todos los involucrados son sujetos de conocimiento. En el marco de esta relación de igual a igual se privilegia las voces de sabios y sabias locales quienes a través de métodos participativos se expresaron abierta y libremente.

La clásica división de roles entre investigadora e informante con la finalidad de obtener datos objetivos, cuantificables o hipótesis verificables, y los valores propios de la investigación clásica pasan a un segundo plano. La dinámica que moviliza los procesos grupales abiertos es el flujo de Acción - Reflexión - Acción. Estos ocurren mediante métodos interactivos de los que surgen ideas, recuerdos, valores, sentimientos que se formalizan en representaciones gráficas. La reflexión grupal contribuye a convertirlos en fuente de inspiración de iniciativas y acciones 
personales y colectivas. El flujo de Acción - Reflexión - Acción conduce al empoderamiento de la comunidad, es decir, a ver la realidad de una forma inédita y enriquecer los horizontes de acción para alcanzar la plenitud de la vida (FALS, 1989; De SOUSA, 2004).

En el Primer Congreso Internacional de Terrazas, en Mengzi, Prefectura de Honghe, China en 2010, las minorías étnicas del Río Rojo, Yunnan, en el Suroeste de China cumplieron un rol protagónico presentando sus terrazas como parte de las actividades del evento (PETERS, 2012). En 2013 aceptamos la invitación del Centro del Patrimonio Cultural de Ifugao que organizó una serie de entrevistas con los guardianes de las terrazas de Ifugao en la Cordillera, Filipinas, como una actividad posterior al primer congreso. Ese mismo año realizamos también una estancia de trabajo de campo con un equipo de jóvenes investigadores y promotores del agro andino, en la comunidad campesina Quechua de Mollebamba en Apurimac, Perú. ${ }^{1}$

El marco del Tercer Congreso Internacional de Terrazas en 2016 sobre la «Búsqueda del Futuro» en Venecia y Padua, Italia, fue ocasión para celebrar diez seminarios en diez zonas locales a lo largo de Italia en los cuales diferentes actores tuvieron el espacio de expresar sus voces. Los grupos de trabajo de campo se reunieron a su regreso en el Horto Botánico de Padova y discutieron sus propuestas de acción resumidas en el Manifesto ITLA (AlBerTI, 2018).

Con motivo del Cuarto Congreso Mundial de Terrazas y Bancales en Las Islas Canarias, Madeira, Azores, Cabo Verde en 2019, se llevaron a cabo una serie de actividades preparatorias, entre ellas una academia de verano y seminariostalleres de IAP en varias islas seleccionadas. Durante el Congreso, los grupos de congresistas se acercaron a los múltiples procesos de «re-encantamiento de bancales» en siete islas (Gran Canaria, La Gomera, Tenerife, El Hierro, La Palma, Lanzarote, Madeira) con la idea de recuperar la integración con la naturaleza para seguir siendo humanos trascendentes (Berman, 1981). Cuatro temas orientaron la elaboración de propuestas de acción: Habitar, Aprender, Saber y Construir en las Terrazas (ITLA, 2020).

\section{LA AGRICULTURA, FUENTE DE CONOCIMIENTOS}

La agricultura surgió paralelamente en distintos puntos del mundo, entre diez y quince mil años atrás, cuando la faz de la tierra entraba en su fase Post Glacial (COWAN y WATSON, 1992). Se le conoce también como la revolución neolítica (SмiтH, 1994), es decir la gradual y milenaria transición de las maneras de pensar y de vivir de las sociedades de cazadores-recolectores creando nuevas condiciones de vida. Entre ellas, destacaron los procesos sociales de ampliar el uso de las variedades de plantas silvestres mediante la domesticación. De esta manera se sentaron las bases de nuevos modos de alimentación contribuyendo

\footnotetext{
1 Sobre la aplicación de la IAP en la comunidad campesina de Mollebamba en 2013 existe un video participativo, la primera parte se puede ver en https:/ / www.youtube.com/watch?v=qZCdUw3-VeQ [Consulta: 29/07/2020] y la segunda parte en https:/ / www.youtube.com/watch?v=2sn19HsCE1Y [Consulta: 29/07/2020]. Esta experiencia fue una de las diferentes actividades preparativas al Segundo Congreso Mundial de Terrazas, «Encuentro de Culturas y saberes de terrazas del mundo», en Cusco, Perú realizado en 2014. Este contó con la participación de una numerosa delegación campesina andina en los distintos espacios de interacción del evento (Tillmann y Bueno de Mesquita, 2015).
} 
a la formación de las sociedades agrícolas. Estos cambios en diferentes sitios del mundo y en múltiples facetas tienen como actores principales a los grupos humanos que en base a la observación sistemática del entorno pusieron en práctica conceptos, valores, normas, construidas socialmente, organizándose en formas de cooperación colectiva para alcanzar el bien común en las más distintas formas culturales (CHILDE, 1956; HAVELOCK, 1986).

Los distintos procesos de desarrollo agrícola revelan una capacidad extraordinaria para construir instrumentos, extensiones de la mano para modificar la naturaleza con imaginación e ingeniosidad. Las sociedades agrarias crearon un repertorio particular de herramientas como las piedras talladas, la fabricación de arados y rastrillos y para ello se basaron en los fundamentos de la geometría, las matemáticas, técnicas para medir y principios de cómputos para evaluar las cosechas. Estos avances tecnológicos y prácticos constituyen la base fundamental de los logros científicos de la humanidad (Eco y Zorzoli, 1961).

Las terrazas agrícolas forman parte del gran mosaico de paisajes emblemáticos asociados a un alto nivel de desarrollo de la agricultura de las zonas montañosas del mundo. Destacan, por su extensión y antigüedad los situados en Asia (China, India, Japón, Corea), en África (Etiopía), en el Mediterráneo, los Andes y Mesoamérica (SANDOR, 2006; TOLEDO, 2009). En estas regiones, los saberes locales atribuyen múltiples significados a las pendientes escarpadas. A veces se les arrogan carácter de divinidades protectoras o fuerzas a ser tranquilizadas, en otras culturas consiste en una relación más bien fraternal. En cualquier caso, las montañas se mantienen enraizadas en las mentes colectivas como símbolos de la naturaleza que merecen respeto y veneración mediante la realización de rituales (EINARSEN, 1995).

Los constructores de terrazas, guiados por la ética de respeto a la naturaleza crearon originales y multifacéticos manejos de procesos geomorfológicos, usos del suelo, del labrado y la construcción de la piedra, del manejo del agua, del aprovechamiento de la variabilidad de clima, de la decodificación de los cuerpos celestes, de la reproducción de plantas por semillas. La fabricación de herramientas manuales merece una mención especial por su notable versatilidad. Los aperos agrícolas en las terrazas de los Andes y Mesoamérica fueron elaborados con gran inventiva, a la medida de las personas y de acuerdo a cada fase de los métodos de cultivo poniendo atención al carácter individual de las plantas (DonKIN, 1979).

A partir del análisis combinado de la antigüedad (SANDOR, 2006), de las denominaciones locales que reciben las terrazas agrícolas (Murtas en AlBerTI, 2018), los cultivos originales más representativos y los que aún se practican en la actualidad, obtenemos un somero inventario de la diversidad de las terrazas agrícolas en el mundo.

\subsection{Asia}

En las montañas del este asiático, en las laderas del río Yangtse se domesticó el arroz, y miles de variedades de arroz se vienen cultivando desde 5,000 y 7,000 años atrás (Fuller, 2016). Las terrazas de agua se llaman shuidao titian en chino. En las montañas Ailao, Yuanyang, Yunnan del Sur, China, las monumentales esculturas de barro llamadas shade, desha en lengua Hani, siguen productivas con 
las tradicionales variedades de arroz desde hace 1200 años atrás, a la par de más de 150 especies de plantas cultivadas en la actualidad (BOUCHERY, 2010).

Las terrazas de arrozales, llamadas tanada en japonés, registran una antigüedad de 2.000 años. También tienen una versatilidad de usos como el cultivo del wasabi (Eutrema japonica), el cerezo y otros frutales así como pozas de piscicultura (FAO, 2018). El uso sostenido de la producción de arroz en las tanada se da gracias a un pacto social entre la población urbana y rural por el cual ciudadanos subvencionan con trabajo y dinero a los productores rurales (Kieninger, 2011). También se mantiene la tradición de áreas de terrazas que producen arroz para el consumo exclusivo del emperador y su familia.

Las altas montañas del Himalaya de Bután continúan cubiertas de bancales con riego y de secano con cientos de variedades de arroz desde hace 3.000 años entre otros miles de especies endémicas (PHuntsho, 2103). Los pueblos del este, los Sharchops usan el término ahring; los Ngalops, del oeste de Bután ahree; y en el sur los Lhotshamps las llaman khaet. $^{2}$

Las terrazas de arroz con riego y de secano reciben el nombre genérico lantangan en lengua de los Ifugao en la Cordillera de Filipinas en el sur este asiático. Tienen una antigüedad de 2.000 años. Desde entonces los Ifugao lograron un complejo y sofisticado paisaje de terrazas compuesto de ocho zonas funcionales que interactúan de forma complementaria. Proveen más de 600 plantas de múltiple uso humano (ConKLIN, 1980). Los Ifugao están revalorizando su conocimiento para la preservación de sus terrazas de arroz a través de organizaciones locales (Guimbatan, 2006).

Las terrazas de Yemen datan aproximadamente entre 5.000 y 6.000 años atrás. Fueron construidas en las empinadas áreas de las montañas Sarawat, con el nombre de marbid. En estas escaleras monumentales se cultivaron cereales, sorgo, trigo y cebada. Ahora siguen en uso con la producción de qat (Catha edulis), un arbusto con propiedades estimulantes para consumo interno y café para exportación (WEIKENMEIER, 1994).

\section{2. África}

El origen de las terrazas en la región de Konso, Etiopía nos remonta 2500 años atrás; se les conoce con los nombres de kawwatta y xeeranta por sus cultivos de cereales y actualmente de café (WATSON, 2009).

\subsection{Europa}

Las terrazas, terrazamenti en italiano tienen más de 30 nombres locales como pastini, vaneze, campigoi, fratte, sgrebeni, loc, massiere, scansie, more, fasce, fasse, canti, cian, tabie, proes, tape, bari (Murtas en AlBERTI, 2016). La civilización pre-helénica del olivo y la vid en el Mediterráneo tienen una antigüedad de más de 3600 años y siguen dando novedosos impulsos económicos a las regiones especializadas en estos cultivos (VAROTTO, 2019).

2 Comunicación personal de Sonam Tashi, agrónomo de la Universidad Real de Bután, coorganizador del Quinto Congreso Mundial de Terrazas a realizarse en 2023. 


\subsection{América}

En los Andes Centrales de América los primeros andenes, pata pata, mullupata en lengua quechua, takuana, patachana en Aymara, se construyeron entre 2500 a 4000 años atrás para domesticar la papa, el maíz, la quinua y cientos de variedades alimenticias. Un complejo manejo de calendarios agrícolas de control estatal sobre la base de las observaciones astronómicas permitió que la civilización andina alcanzara su apogeo en la época incaica (EARLS, 2015; COOK, 2011; ZUIDEMA, 2010).

En las montañas de Mesoamérica, cuna de las civilizaciones Maya y Azteca se cultivaron variedades de maíz en terrazas irrigadas del valle de México y datan más de 3000 años atrás. Actualmente los campesinos denominan a las terrazas con el nombre local de meteplante, una voz derivada de la lengua Náhuatl (Pérez, 2014), y en castellano se les conoce como trincheras.

A lo largo de los siglos la historia de la agricultura, las sociedades han dado lugar a procesos de acumulación de valiosos cuerpos de experiencias colectivas transmitidos oralmente de generación en generación y mediante entelequias culturales. Esta riqueza cognitiva de los pueblos enfocada desde las minuciosas y precisas formas de percepción de los fenómenos naturales, en especial de plantas y animales, confirman que los pueblos que viven y producen lo hacen empleando los atributos universales del pensamiento humano. Estos saberes no son fruto del azar, ni únicamente de la necesidad material, tampoco de la ignorancia o la irracionalidad. Cada grupo humano analiza, distingue y clasifica los fenómenos naturales con categorías y significados propios en sus idiomas, a veces difíciles de traducir (LeVI StRAuss, 1964).

Desde la década de los 80 contamos con estudios efectuados por diferentes disciplinas científicas conocidos como Conocimiento Técnico Indígena (ITK por sus siglas en inglés). Se recopilaron un amplio repertorio de las capacidades tecnológicas de los pueblos sobre el manejo del territorio, sistemas de producción agrícola, prácticas de salud, de la alimentación y otros campos del conocimiento de grupos humanos considerados no occidentales (BROKENSHA et al., 1980).

El enfoque antropológico e interdisciplinario de los saberes de los pueblos se complementa con el análisis de colecciones botánicas en todo el mundo que han reconocido áreas geográficas donde se concentran miles de variedades de especies de plantas. Estas variedades coinciden con territorios habitados por grupos humanos, quienes dieron lugar a los ocho centros de domesticación (VAVILOV, 1992), la mayoría en zonas montañosas, la cuna física de las sociedades que inventaron los paisajes de terrazas para vivir cultivando alimentos.

Llegamos a los estudios actuales de la biodiversidad que consideran que, para ser, vivir y producir, el mundo cuenta con la gran riqueza de 2.1 millones de variedades de plantas y existen más de 7000 especies domesticadas a lo largo de la historia de las sociedades agrícolas (Biodiversidad, 2018; ETC, 2017). De la misma manera los estudios lingüísticos han identificado más de seis mil lenguas en el mundo que revelan más de cuatro mil formas de vida con finas expresiones estéticas, de valores culturales y espirituales particulares imbricados en multifacéticas cosmovisiones del mundo (Harmon en MAFFI, 2001; POSEY, 1999).

La perspectiva de los Sistemas de Conocimiento (Marglin, 2000) nos permite entender los saberes en sus formas particulares y en términos de cuatro características: 
1) La epistemología, trata sobre las explicaciones que los grupos humanos dan a sus saberes en sus propios términos conceptuales y considerando las numerosas variaciones de los sujetos de conocimiento.

2) La transmisión del saber, se refiere a cómo se comunican las ideas entre los grupos y las generaciones, siempre distinguiendo los distintos saberes, así como las diversas modalidades de comprender el conocimiento.

3) La innovación, es una propiedad de todos los cuerpos de conocimiento que ocurre por diferentes razones, endógenas y exógenas, bajo condiciones muy particulares difíciles de generalizar. Afirmar que los saberes son tradicionales, que sus corpus son estáticos debido a las reglas fijas de las costumbres desatiende la dinámica intrínseca del saber.

4) Finalmente, el poder se refiere a quién define las relaciones que configuran la generación y el uso del conocimiento dentro del sistema y en interacción con otros sistemas de conocimiento.

Abordar los saberes agrícolas con el conjunto de estas miradas incluye abrazar las ideas simbólicas del espacio y del tiempo, los conceptos sagrados que dan forma a la creación de paisajes locales (GAVAZZI, 2010) y poner especial atención a los valores espirituales en la organización del trabajo (BOUCHERY, 2010). También significa enfocar los ingeniosos métodos para conservar los alimentos y las maneras exquisitas de disfrutar la comida, así como los más diversos y entrañables sentimientos de identidad con las plantas, con los paisajes y territorios donde se entrelazan la vida y la producción (USMP, 2005; SALAS, 2013). Cabe resaltar los saberes diferenciados entre hombres y mujeres, entre jóvenes y los mayores de edad, depositarios de la sabiduría.

De los estudios interdisciplinares que enfocan los sistemas agrícolas y el medio ambiente, las plantas alimenticias y medicinales, cultivadas y silvestres reconocemos la vigencia de un rico repertorio de prácticas locales. Estos son los pilares de la sostenibilidad de las sociedades rurales ya que cumplen con el ideal del bienestar colectivo (Prain et al., 1999; SANTOSOMBAT, 2002).

Las sociedades agrarias y sus saberes plurales nos indican la vigencia de la inextricable relación con la naturaleza. Estas no responden a un solo molde de pensamiento sino a la consciencia de la diversidad que son múltiples e incesantes, complejos y dinámicos aprendizajes sociales. Esta complejidad está contenida en el concepto de la diversidad biocultural, la relación entre los miles de lenguas habladas en el mundo, los saberes y el medio ambiente (MAFFI, 2001; LAIRD, 2002).

El aporte histórico y productivo de los saberes creadores de espacios para vivir y producir en diversidad conduce a visualizar un dilema de dimensión global, es decir, la actual relevancia y la fragilidad de los paisajes de terrazas. La relevancia, radica en que las terrazas forman parte del conjunto de sistemas agroalimentarios locales, de pequeña escala que producen una gran variedad de cultivos originarios para la alimentación global (KHOURY, 2016). La fragilidad, consiste en que los agricultores, las agricultoras de terrazas, como parte de los productores, las productoras de alimentos, son objeto de una creciente amenaza de sus formas de producir y de vivir y con ello se afecta la continuidad de la vida en el planeta (Figura 1).

Este peligro proviene de un complejo escenario científico y de poder político que defienden la agricultura industrial como la panacea que alivia el hambre mundial, cuando en realidad su impacto sobre el ambiente, las sociedades y los saberes locales es funesto (KIMBRELL, 2002). A nuestro parecer la siguiente interfaz 
es uno de los puntos clave de este nudo crítico. De un lado tenemos que, en términos poblacionales, los sistemas agroalimentarios se componen de tres mil millones de pequeños productores (hombres, mujeres), familias, comunidades campesinas, agricultores urbanos, pastores nómades, pueblos indígenas, pescadores artesanales, recolectores del bosque, que accediendo al 30\% de los recursos de tierra proveen a la humanidad con el $70 \%$ de los alimentos de la amplia gama de agrobiodiversidad. Mientras que la agroindustria accede a más del $70 \%$ de los recursos agropecuarios del mundo para alimentar a menos del 30\% de la población mundial con productos de reducida agrobiodiversidad. Además, utiliza al menos $90 \%$ de combustibles fósiles dejando una huella de destrucción ecológica de gran magnitud (ETC, 2017).

Los paradigmas de conocimiento dominantes que fomentan el modelo agroindustrial moderno se sustentan en las tecnologías financieras, en las cadenas de bloques y en las plataformas de datos masivos. Estas se manejan desde algunos laboratorios en los centros científicos controlados por los grandes consorcios del sector agrícola y alimentario. Acceden a información digital de, por ejemplo, plantas conservadas genéticamente en cualquier parte del mundo y con asombrosa rapidez construyen diseños genéticos a la medida exacta de los intereses económicos de la agroindustria (MOONEY, 2019). La acción política fortalece el conocimiento de quienes controlan las plataformas de datos en manos de muy pocas personas al servicio de los grandes y poderosos consorcios de la agrotecnología. De esta manera se subordinan los saberes que nacen de la dinámica, la complejidad y la diversidad de los ecosistemas causando el epistemicidio (DE SOUSA, 2014). Es decir, una injusta exclusión de los saberes plurales por un modelo único de conocimientos que se considera epistemológicamente superior. Esto amenaza gravemente la sustentabilidad de la vida social en el mundo privándonos del derecho a vivir diversas formas de vida y al ejercicio de la soberanía alimentaria que contempla de manera crucial las decisiones de los agricultores sobre la calidad de la producción y con qué saberes producir los alimentos (PIMBERT, 2018).

Por ello, se hace imperativo que los singulares repertorios de saberes de los grupos humanos que comparten lenguas, conceptos, valores y prácticas en sintonía con la naturaleza recuperen y recreen la memoria biocultural para superar las encrucijadas de la crisis moderna (TOLEDO y BARRERA-BAsSOLS, 2008).

\section{RESULTADOS}

\subsection{Ideas, conceptos, valores, prácticas y visiones de sabios y sabias de terrazas}

Según FALs (1992) la presentación de resultados de procesos IAP es una manera de amplificar la construcción conjunta de sentido, fruto del diálogo de saberes y se elabora en tres niveles comunicativos. El primer nivel de devolución consiste en la comunicación de los resultados visuales generados por los grupos con la finalidad de que estos se reconozcan y emprendan las acciones de recuperación históricocultural de sus visiones colectivas de una manera autónoma. El segundo nivel de devolución comprende el intercambio sistemático de experiencias documentadas con la finalidad de enriquecer el bagaje metodológico y la consciencia participativa de los investigadores comprometidos con las transformaciones populares. El tercer nivel de devolución incluye la elaboración de artículos o libros tratando 
temas específicos que proponen una reflexión metodológica con la finalidad de ampliar el horizonte teórico de la praxis participativa.

En este artículo nos inscribimos en el tercer nivel de devolución de los resultados. Nos basamos en las expresiones subjetivas que surgieron de las diferentes formas de interacción dialógica con sabios y sabias en el contexto de los cuatro Congresos Internacionales de paisajes de terrazas en el transcurso de diez años. Nuestra intención es abrir un resquicio de reflexión intercultural sobre la diversidad de los saberes locales que poseen quienes producen y viven en paisajes de terrazas.

Para ello nos valemos de veinte representaciones visuales acompañadas de textos. Estos pasajes escritos responden a nuestra recepción de los testimonios orales de quienes los generaron. Redactamos los contenidos valiéndonos de la memoria y de las anotaciones personales al momento que fueron expresados en el contexto de los Congresos Internacionales en un esfuerzo comunicativo, hilvanando con respeto la autenticidad del testimonio. En el caso de Yuanyang (China) y de Ifugao (Filipinas) nos respaldamos en las traducciones del idioma local al inglés para redactar las versiones en castellano.

La selección de representaciones gráficas está estructurada en cuatro categorías: el espacio, el tiempo, los saberes y las visiones de futuro, generado por sabios y sabias a través de métodos participativos como mapas, perfiles, diagramas, ciclos, calendarios, dibujos, matrices y biografías. En primer lugar, presentamos una gama de percepciones e ideas locales sobre el espacio, incluyendo las zonas donde se ubican las terrazas, qué y dónde se produce, los diferentes tipos de terrazas, así como los parajes donde acontece la vida social. En segundo lugar, mostramos representaciones que abordan diferentes aspectos relevantes a las actividades agrícolas en terrazas en el transcurso del tiempo. En tercer lugar, abordamos ejemplos de cómo se valoran los saberes por sus propios actores y qué reconocimiento externo reciben. Finalmente desplegamos las visiones que nutren las aspiraciones a la plenitud de la vida, entendidas en muchas formas culturales, como por ejemplo el Sumaq Kawsay (Buenvivir) de los pueblos quechua (GudynAs, 2011; Acosta, 2010) de los Andes.

Cada una de las representaciones es un fulgor micro cósmico de la complejidad y profundidad de las entelequias que mantienen vivas la producción en las terrazas. Son aportes cognitivos de diferentes culturas, indispensables en la construcción de los espacios democráticos y de transformación creativa para una vida sostenible (DE SOUSA, 2004).

En varios textos sugerimos ver videos para familiarizarse con el ambiente, el paisaje, la gente en referencia, de una manera más amplia.

\subsection{El Espacio}

\subsubsection{La riqueza de mi tierra}

Silco, barrio de la comunidad campesina de Mollebamba, Apurimac, Perú (Figura 2). Los autores de esta representación nos muestran la empinada ladera donde viven y producen, dividida en tres zonas. En la zona alta a más de $4900 \mathrm{msnm}$ residen los Apus (montañas sagradas) que protegen los territorios comunales. La presencia del cóndor indica la sacralidad del lugar. Allí vive la vicuña que se 
alimenta de ricos pastos naturales. A 3500 msnm comienza la zona media donde se encuentra el reservorio de agua que se llena con las aguas nacientes provenientes de las cumbres nevadas. Los canales irrigan las áreas donde crecen la cebada y los cereales andinos kiwicha, cañihua. Allí se agrupan unos cuantos árboles de quinual, quishuar y aliso. Debajo de los $3000 \mathrm{msnm}$ comienza la parte baja donde se ubica el pueblo y viven las familias campesinas. Otro reservorio lleva agua a las terrazas de maíz, así como distintas variedades de papas, ocas, mashua, olluco y quinua. Alrededor de las viviendas están los corrales de las vacas, cerdos, ovejas, gallinas y patos que se crían para el consumo familiar.

Este orden del territorio de terrazas tiene raíces en la cosmovisión andina. «Hanan Pacha», el mundo de las eternas alturas, «Kay Pacha», el mundo presente de la vida social en comunidad, $\mathrm{y}$ «Uku Pacha», el mundo pasado. Estos tres mundos están interconectados de manera inseparable, en ellos se entrelazan el flujo del agua, la organización de la ganadería, la vida social y el manejo de la diversidad de los cultivos bajo el manto protector de la montaña sagrada. Los comuneros valoran esta manera de vivir con una vida plena. Se sienten amenazados por el perjudicial impacto ecológico de la industria minera que socaba y contamina los territorios donde nacen los acuíferos. Ven con mucha tristeza que la demanda de mano de obra en la minería rompe los lazos solidarios y de ayuda mutua para mantener los andenes productivos. ${ }^{3}$

El Programa Andino de Cambio Climático (PACC) elaboró un video participativo sobre la microcuenca de Mollebamba, ambientado en el paisaje de terrazas y con las voces originales de la comunidad. ${ }^{4}$

\subsubsection{Los cultivos y las plantas silvestres}

Yuanyang, Prefectura de Honghe, Provincia Yunnan, PR China (Figura 3). Las terrazas de Yuanyang en Yunnan de una antigüedad de 1000 años se ubican en las laderas de las montañas Ailao. Es un territorio multiétnico que comprende cinco zonas agrícolas. Según las dos campesinas Yi graficaron el uso de las terrazas desde las cumbres boscosas a los 1500 msnm donde viven los Hmong. Este grupo étnico se responsabiliza por el mantenimiento de las fuentes de agua asegurando el flujo por los canales para que las terrazas nunca se sequen. Descendiendo en dirección hacia el Río Rojo (300 msnm), cada grupo étnico Hani, Yi, Yao, Zhuang y Dai que posee su propia lengua y cultura, vive y produce en las siguientes zonas: los campos de agricultura rotativa, las terrazas y los campos en secano y los famosos arrozales inundados. La comunicación entre las etnias es posible pues comparten el derecho consuetudinario con el presupuesto moral de mantener en equilibrio las laderas. Se inspiran en el concepto de unidad entre la comunidad humana y los dioses. Entre las etnias prima un reconocimiento social por específicas habilidades prácticas y espirituales de producir en diversidad para el bien social (Presentación de dos custodias de terrazas en el Primer Congreso de Terrazas, Mengzi, Río Rojo, China RP). ${ }^{5}$

3 Raúl Vera de 48 años y su hijo Kevin Vera de 12 años, Taller comunal de terrazas en Mollebamba, Apurimac, Perú 2013.

4 Youtube: https:/ / www.youtube.com/watch?v=2uctE1xYvVk [Consulta: 29/07/2020].

$5 \mathrm{El}$ video producido por la TV China nos ofrece una vista panorámica de las terrazas de Yuanyang: https://www.youtube.com/watch?v=hD3hcNe_nM4\&feature=emb_logo [Consulta: 29/07/2020]. 


\subsubsection{El valle cubierto de olivos}

Temisas, Gran Canaria, España (Figura 4). Esta representación de un vecino mayor notable de Temisas nos muestra un panorama en el que se entretejen los bancales y el pueblo. Es un paisaje que se encuentra estructurado en tres barrios, por sus barrancos: El Henchidero con las escolleras, El Centro (Juncal Alto) con los bancales que se construyeron para deshacerse de la piedra, y El Callejón con las cadenas de riego y secano. Hasta hace 50 años atrás el agua corría por estos barrancos y era distribuida por el sistema de las dulas, dando vida al cultivo de olivos en el valle y a las cadenas con riego que abarcan las laderas en la parte alta del pueblo. Se producía cereales y papas para alimentar a más de 800 habitantes. Se contaba con profesionales pedreros, que construían muros de piedra, rancheros, que distribuían el agua, cesteros que tejían canastas con hojas de palma y molineros que manejaban los molinos de aceite. Ahora hay menos de 350 habitantes, la mayoría es de la tercera edad. El pase generacional de los saberes agrícolas se ha interrumpido porque los jóvenes han abandonado la agricultura y la ganadería por el empleo que genera el turismo de la costa. Las pocas familias jóvenes establecidas en Temisas están revitalizando la producción de olivos y la gastronomía local. ${ }^{6}$ El video sobre Temisas nos muestra las cadenas antiguas y el paisaje reflejado en el dibujo. ${ }^{7}$

\subsubsection{Bancales en función y bancales abandonados}

Arbejas y Juncalillo, Galdar, Gran Canaria, España (Figura 5). En Arbejas y Juncalillo, los pobladores reconocen dos tipos de terrazas. Unas con «apoyo» (trabajadas por pobladores) y otras «abandonados» (en retroceso y sin uso). En las Arbejas el paisaje de terrazas con «apoyo» muestra cuatro zonas diferenciadas por la altura y tipos de cultivo y el manejo de agua. La parte más alta es una pendiente muy fuerte. En los bancales por encima de la casa cueva crecen cereales. Siguiendo en descenso en cada plataforma se cultivan diferentes árboles frutales, manzanos, almendros, higos. En los bancales más bajos se cultiva millo (maíz) y papa. En la base del sistema de terrazas un reservorio de agua al mismo nivel del barranco lleva el nombre de Arbejas.

En Juncalillo los bancales de secano anteriormente cultivados están en abandono o en uso extensivo. Una fina percepción de la vegetación indica los años de abandono de los bancales. Los árboles más altos como el castaño y el nogal rodeados de malezas muestran que el abandono data de 10 años o más. Donde crece el tagasaste los bancales están abandonados desde hace 8 años (aunque el tagasaste es usado para el ganado), donde crece el incienso se abandonaron hace 4 años y donde crece la avena fatua desde hace 2 años. En la base de los bancales se encuentra el Barranco Hondo. ${ }^{8}$

6 Don Miguel Jiménez, de Temisas, Gran Canaria, taller de preparación del Iv Congreso Mundial de Terrazas, 2019.

7 Youtube: https:/ / www.youtube.com/watch?v=96zE0BZaX1U [Consulta: 29/07/2020].

8 Juncalillo, Gran Canaria, taller de preparación del Iv Congreso Mundial de Terrazas, 2019. 


\subsection{El Tiempo}

\subsubsection{Calendario Sumaq}

Mollebamba, Apurimac, Perú (Figura 6). El período clave de este ciclo anual es junio, el año nuevo andino, cuando aparecen las Pléyades en el cielo de la madrugada. Según las explicaciones de un anciano, una mujer comunera y un joven refieren que la intensidad del destello de este cúmulo de estrellas indica que será un buen año, si las estrellas no brillan será todo lo contrario. La luna de julio y agosto en sus distintas fases anuncia cómo será la temporada de lluvias.

En setiembre y octubre hay que observar la vegetación y los frutales. En noviembre se advierte la conducta de ciertos animales. Cada comunidad, cada familia, tiene su propia manera de descifrar los mensajes de las estrellas, la luna, el sol además de otros indicadores de la naturaleza y de esta manera se programan las actividades correspondientes a cada cultivo.

El título de este calendario es sumaq en quechua, significa 'bueno', 'bello'. Son los valores con los que la memoria colectiva descifra el comportamiento de los fenómenos celestiales. La finalidad de organizar las actividades agrícolas marca significativos momentos técnicos y a la vez rituales a lo largo del año que se celebran con la presencia de tres generaciones. Las celebraciones rituales dentro del calendario agrícola afirman el trabajo como una actividad dignificante y no como un lastre. ${ }^{9}$

\subsubsection{El calendario de la diversidad de papas}

Los Realejos, Tenerife, España (Figura 7). Esta representación elaborada por los miembros de La Asociación de Papa Bonita de los Realejos muestra la organización de las labores agrícolas de múltiples cultivos como la papa, la vid, las judías, los ajos, los millos, el trigo, y los frutales, incluye también actividades ganaderas y la artesanía. Los detalles nos revelan una lógica secuencial que toma en cuenta una complejidad de factores interdependientes como las zonas agrícolas, el tiempo (semanas, meses), la aparición de las lluvias, la rotación y el tipo de cultivo, la maduración de los frutales, la celebración de fiestas religiosas y el mercado. Todos ellos condicionados a diversas contingencias que tiñen el calendario de un sofisticado relativismo. ${ }^{10}$ Véase el sitio web de la asociación para mayor información sobre la historia, la producción y las variedades de papa. ${ }^{11}$

\subsubsection{Entre siembras y cosechas}

Juncalillo, Gran Canaria, España (Figura 8). El calendario agrícola, aunque

\footnotetext{
9 Don José Silvestre, 77 años, Elena Neyhery León de 50 años y Leoncio Huarcaya de 15 años, en el taller comunal sobre terrazas en Mollebamba, Perú 2013.

10 Los Realejos, Tenerife, preparación del Iv Congreso Mundial de Terrazas, 2019.

11 La papa bonita [en línea]. Disponible en: www.lapapabonita.com [Consulta: 29/07/2020]. Los siguientes videos sobre los Realejos muestran el paisaje y el trabajo agrícola en terrazas: https:// www.youtube.com/ watch?v=SCtae0Xw8aE [Consulta: 29/07/2020]; https://www.youtube.com/ watch?v=WM3ABY4MBVg [Consulta: 29/07/2020].
} 
formalmente es un listado de actividades, tiene como hilo conductor la idea de la continuidad cíclica. El punto de partida es setiembre, final del verano y a la vez el reinicio de las actividades. Es un complejo ciclo, elaborado por un agricultor, en el que cada período refleja penetrantes y sensoriales observaciones de la lluvia, del frio, de la floración de los frutales, el calor, la sequedad del ambiente factores que se relativizan para el manejo de varios cultivos a la vez. El calendario revela un razonamiento comparativo entre los matices de las categorías climáticas y destaca los momentos propicios. ${ }^{12}$ Los videos que recogen las voces de mujeres y hombres de Juncalillo (parte del Patrimonio Mundial de UNESCO del Risco Caído y las montañas sagradas de Gran Canaria) nos acercan al paisaje y la vida en Barranco Hondo. $^{13}$

\subsubsection{Cambio climático}

Mollebamba, Apurimac, Perú (Figura 9). Este gráfico que trata sobre la lluvia, el agua y los andenes en los últimos cincuenta años. Nos presenta a la lluvia cuando era parte armónica del mundo natural. El agua corría libremente y regaba los andenes que estaban bien conservados y productivos. Desde hace cincuenta años la lluvia se ha moderado y el agua ya no colma los reservorios que riegan los andenes donde crecen los cultivos alimenticios. Desde hace veinte años las lluvias son muy irregulares, el caudal del riachuelo está disminuyendo y algunas paredes de piedra están venciéndose. Hoy en día las lluvias se han vuelto destructivas, las aguas merman y las paredes de los andenes se caen sin que se puedan reparar.

Estos recuerdos de un campesino y una campesina mayores correlacionan tres aspectos del paisaje con sus vidas como agricultores. La lluvia, el agua y los andenes les plantea la experiencia del cambio climático como una ruptura del orden natural. Este sentir inquietante es compartido por comuneros y comuneras en muchas partes de los Andes Centrales que buscan explicaciones y formas de restaurar la armonía con la naturaleza (TillmanN, 1997). ${ }^{14}$

\subsection{Saberes, profesionales y especialistas}

\subsubsection{La abundancia de las plantas silvestres en las terrazas de arroz}

Ifugao, Cordillera, Filipinas (Figura 10). La maestra de escuela de Hungduan en Ifugao, Filipinas, es una gran conocedora de las plantas (Figura 10 izqda.). En un recorrido de 45 minutos por las terrazas de arroz, recogió más de 60 plantas en su mayoría silvestres. Las clasificó por sus propiedades alimenticias, medicinales, fertilizantes y espirituales. Con la ayuda de varones mayores identificaron los nombres locales y recordaron cómo antes se recogían esas plantas durante el período de descanso de los cultivos, evocaron qué platos se preparaban, cómo se curaban con ellas y reconstruyeron el significado de cada una de las plantas

12 Elaborado por Julián de Juncalillo, Gran Canaria, taller de preparación del Iv Congreso Mundial de Terrazas, 2019.

13 Youtube: https://www.youtube.com/watch?v=1MQSsINGLfQ [Consulta: 29/07/2020]; https:// www. youtube.com/watch?v=xzgdoJKUt5I [Consulta: 29/07/2020].

14 Josefina y Aurelio Dongo, de 65 años, en el taller comunal de terrazas de Mollebamba, Perú 2013. 
en las diferentes celebraciones rituales (Figura 10 dcha.). Ante la presencia de extensionistas agrícolas de la localidad de Banaue y algunos jóvenes, la colección de plantas generó un debate sobre el valor de los alimentos. Valoraron la abundancia de aquellos alimentos que provienen de las terrazas sin ser cultivados, reconociendo los sabores, lo saludable y la independencia económica asociada al consumo de productos locales. Compararon el negativo impacto de la alimentación agroindustrial en la salud de las personas, en el medio ambiente y por la dependencia del dinero para adquirir los productos.

El reconocimiento de la abundancia de plantas es una práctica relacionada a la manera de entender y gestionar el espacio como un todo complejo compuesto de ocho componentes, o zonas agroecológicas que el pueblo Ifugao mantiene en su memoria colectiva. En conjunto, el sistema sintoniza finamente los ciclos de la naturaleza, la conservación de la tierra, el control de pestes y enfermedades y la diversidad de plantas domesticadas y silvestres. Los agricultores Ifugao reconocen las cualidades especiales de las rocas, el agua y otros elementos naturales en el uso respetuoso de los recursos. Estos valores se reafirman en la continua celebración de rituales agrícolas en cada fase de un sofisticado calendario agrícola a cargo de la figura del Mumbaki. (Conklin, 1980; Trabajo de campo en Hungduan, Ifugao, Filipinas 2013)

Las terrazas de arroz han ganado el merecido reconocimiento de la UNESCO como Patrimonio de la Humanidad desde 1995 confirmando la importancia de los saberes del pueblo Ifugao. Sin embargo, el turismo de masas y las políticas de modernización agraria privilegian demandas mercantiles y desequilibran la sostenibilidad de este sistema agrícola que ha llevado miles de años en articularlo a la perfección. En la actualidad el pueblo Ifugao defiende el derecho a seguir viviendo y practicando el complejo y sofisticado conocimiento de su territorio con apoyo de ONGs locales (UNESCO, 2008; GuImbATAN, 2006; BAGUILAT, 2012). ${ }^{15}$

\subsubsection{Maestro Pedrero}

Icod de los Vinos, Tenerife, España (Figura 11). El señor Abdul tiene muchos años de experiencia profesional en el campo y la ciudad. Explica la construcción de muros de piedra seca con las siguientes palabras «Miro el vacío, elijo la forma de la piedra, la modifico y construyo». Para él, la sabiduría y técnica de los muros dependen de la selección de las piedras por su forma, textura y dureza así como el labrado usando diferentes herramientas como el martillo, mandarria, maceta y guataca. El valora el saber y el arte que posee por tradición familiar. Desestima los muros de ahora que se hacen muy rápido, con la ayuda de máquinas y se acaban poniéndoles una capa de cemento. ${ }^{16}$

La profesión de pedrero con la finalidad del mantenimiento de los muros en términos agro-técnicos y estéticos está reviviendo a pesar de la interrupción del pase generacional. Por un lado, la educación formal de Institutos Agrícolas intenta promocionar los conocimientos de la construcción de muros mediante

15 Podemos ver una explicación de las terrazas de arroz en el video sobre Ifugao como Patrimonio de la Humanidad en: https://www.youtube.com/watch?v=ype6GAzREdM\&feature=emb_logo [Consulta: 29/07/2020].

16 Finca Boquín en Icod de los Vinos, Tenerife, taller de preparación del IV Congreso Mundial de Terrazas, 2019 
cursos de capacitación, así como a través de distintas iniciativas de aprendizaje por el trabajo. De otro lado, los impulsos a los pedreros se suman ya que en 2018 la Unesco reconoció la construcción de muros secos en Suiza, Chipre, Grecia, Croacia, Francia, Eslovenia, Italia y España, como Patrimonio Intangible de la Humanidad. ${ }^{17}$

\subsubsection{Minería del agua: entre la vida y la muerte}

Juncalillo, Gran Canaria, España (Figura 12). La historia de vida de Santiago nos muestra a un especialista en excavación de galerías para captar agua de uso agrícola. Nos cuenta su vida en base a hitos naturales. Considera el fuego muy peligroso como en la quema descontrolada que causa incendios en los pinares. Las cabras no encuentran qué comer ni lugar donde protegerse, es la muerte. El agua en cambio es para él fuente de vida, por eso se hizo excavador de galerías. Aprendió a usar el pico y la dinamita. Esta práctica puso muchas veces en alto riesgo su vida; una vez se quedó nueve días metido debajo de la tierra. Don Santiago valora el agua, considera que es vital para la agricultura de la localidad. Recuerda con tristeza que desde los años sesenta, la gente está emigrando y con ella el agua también. Ver que los campos están secos y los pueblos sin gente le lleva a imaginar el futuro como un desierto. ${ }^{18}$

\subsubsection{Mi vida está atada al maíz}

Cabanaconde, Valle del Colca, Arequipa, Perú (Figura 13). Doña Flora, comunera de Cabanaconde, presenta a través de una muestra de variedades de maíz, la sabiduría sobre el uso, manejo, conservación y producción de los andenes que está presente en las manos, cabezas y vidas de las familias y organizaciones comunales. Sobre todo, reconoce que su vida está atada al maíz en toda su diversidad de colores, tamaños y sabores. Ella y los miembros de su comunidad seguirán sembrando maíz, aunque el mercado no reconozca este esfuerzo y diga que no es rentable. Ella resistirá, pero no sabe hasta cuándo. ${ }^{19}$ El Programa del Sur Andino de la ONG DESCO ha producido 3 videos sobre la andenería del Valle del Colca, el primero sobre la agrobiodiversidad, el segundo sobre la reconstrucción de andenes y muros de piedra seca y el tercero sobre los sistemas de riego. En el segundo video habla Flora Chuiquicondor sobre la diversidad de sus variedades de maíz. ${ }^{20}$

17 En referencia al arte de los muros secos, véase: https://ich.unesco.org/en/RL/art-of-dry-stonewalling-knowledge-and-techniques-01393 [Consulta: 29/07/2020]. Véase el video sobre el arte de los pedreros que ganó el segundo premio en el concurso de videos del III Congreso de Terrazas en Italia: https://www.youtube.com/watch?v=ku6f3gz9nRA [Consulta: 29/07/2020].

18 Don Santiago, Barranco Hondo, Gran Canaria, Taller preparatorio del Iv Congreso Mundial de Terrazas, 2019.

19 Flora Chuquicondor, Cabanaconde, Perú en el II. Congreso Mundial de Terrazas en Cusco, Perú, 2014.

20 Youtube: https://www.youtube.com/watch?v=ZrB_TP9T7Fg [Consulta: 29/07/2020]; https:// www. youtube. com/watch?v=57imrhSWv14 [Consulta: 29/07/2020]; https://www.youtube.com/ watch?v=1c2WI9CNk9w [Consulta: 29/07/2020]. 


\subsubsection{Plantas que llegaron y plantas que estaban}

La Gomera, Islas Canarias, España (Figura 14). Dos profesionales, guías del Parque Nacional de Garajonay, La Gomera, graficaron y recordaron un significativo número de plantas y árboles que conocen a través de sus experiencias de trabajo. Emplearon el criterio de las diferentes alturas y la memoria para ubicar la vegetación desde el bosque a 1487 msnm hasta la costa. Emplearon la idea de la clasificación binaria expresadas en dos grandes categorías. Del lado izquierdo están las plantas «que llegaron», como la papa, el plátano, la batata, las verduras y otras plantas cultivadas. Por el lado derecho están las plantas «que estaban» que en su mayoría son las plantas endémicas silvestres. ${ }^{21}$

\subsubsection{El rol de las mujeres en la protección de las terrazas}

Yuanyang, Río Rojo, Yunnan, China (Figura 15). Las terrazas de Yuanyang se manejan gracias al concierto multiétnico de saberes especializados que gozan de reconocimiento social. Las mujeres de la etnia Hani gozan del reconocimiento como las guardianas de semillas, por el especial aprecio que sienten por las diferentes variedades de arroz glutinoso y de diferentes colores. Ellas saben que las prácticas de conservación de varias decenas de variedades locales de arroz dependen del mantenimiento de las terrazas inundadas de agua, del empeño de responsabilizarse por el flujo continuo del agua en las terrazas, así como de la recolección de hojas para fertilizar las terrazas y del secado del arroz al sol. El arroz glutinoso y la diferente gama de colores es la prioridad en el consumo familiar y fuente de su identidad étnica. Por ello, las mujeres Hani son las primeras en resistir pacíficamente frente a la imposición de la política agraria que introduce semillas de arroz híbridas, comerciales. ${ }^{22}$

\subsection{Visiones de futuro}

\subsubsection{Los ingredientes del re-encanto}

Juncalillo, Gran Canaria, España (Figura 16). Esta imagen nos transmite las ideas que se requieren para construir el futuro de Juncalillo, Gran Canaria. Se entiende como un proceso de re-encanto de las terrazas cuyos ingredientes locales y colectivos son la historia, la solidaridad, la naturaleza, la experiencia, sus productos, sus fiestas, su gente y el turismo. ${ }^{23}$

21 La Gomera, enero de 2018, Taller preparatorio del Iv Congreso Mundial de Terrazas de 2019. Amplia información sobre el parque Garajonay puede verse en la página web del gobierno de Canarias [en línea]. Disponible en: http://www.gobiernodecanarias.org/parquesnacionalesdecanarias/es/ Garajonay [Consulta: 29/07/2020].

22 Primer Congreso de Terrazas 2010, Mengzi, Río Rojo, China RP. Las terrazas de arroz de Yuanyang son Patrimonio de la Humanidad de Unesco desde 2013, una ventana de esperanza de apoyo a vivir y producir en diversidad.

23 Taller de preparación del Iv Congreso de Terrazas, Gran Canaria, 2018. 


\subsubsection{Continuidad de la vida en los Andes}

Mollebamba, Apurimac, Perú (Figura 17). Un grupo de niños y niñas quechua representan el futuro de su comunidad con la presencia imponente de las montañas protectoras. Aparecen las diferentes zonas de cultivos andinos, los andenes productivos, los canales y los reservorios de agua. Los niños expresan un paisaje muy parecido al presente sin grandes cambios salvo el tamaño de las casas que da a entender el deseo de seguir siendo campesinos quechua en sus territorios de terrazas con mejores condiciones. ${ }^{24}$

\subsubsection{Del pasado al futuro de la agricultura en andenes}

Moray, Cusco, Perú (Figura 18). Para la juventud del campo cuzqueño el sistema de terrazas de Moray, creado hace 800 años como un centro de experimentación de la diversidad del maíz, se ha convertido en un símbolo de la visión de Soberanía Alimentaria. Este concepto es entendido como la resistencia a la mentalidad extractivista de la minería y de la agroindustria. La juventud rural reconoce los valores de la agricultura andina en terrazas como modelos sostenibles para compartir solidariamente el buen vivir de la sociedad. Expresa sus anhelos de gozar del sentimiento de ser parte del paisaje agrícola, trabajar bajo los principios andinos de la ayuda mutua y ser autónomo en las decisiones sobre qué tipo de agricultura quieren practicar y qué calidad de alimentos quieren producir. $^{25}$

\subsubsection{Identidad}

Isola de Ischia, Italia (Figura 19). La visión de los jóvenes de la Isla de Ischia, Italia fue presentada por Silvia d'Ambra de la Asociación Slow Food. Apuesta a una formación integral en la escuela técnica de agronomía, que les permita reconocer sus profundas raíces de identidad etrusca, romana, fenicia y griega de más de 2.000 años. Ven en el futuro la oportunidad de practicar una agricultura diversificada que garantice la comida de calidad orgánica, plantas medicinales, y vinos ecológicos (TILlmann y VAROTTO, 2016). ${ }^{26}$

\subsubsection{Vivir en armonía con la naturaleza}

San Bartolomé de Tirajana, Gran Canaria, España (Figura 20). Dos mujeres urbanas decidieron transformar sus vidas creando la empresa turística Eco Tara, en Fataga (Gran Canaria), una localidad donde se sienten parte de un paisaje

24 Taller comunal de Terrazas en Mollebamba, Perú, 2013.

25 II Congreso Mundial de Terrazas, Cusco, Perú, 2014. Video explicativo del centro de experimentación de Moray: https:/ / www.youtube.com/watch?v=UeMUZzl4ZEo [Consulta: 29/07/2020].

26 Véanse las actividades del grupo en el blog Slow Food Yschia e Procida [en línea]. Disponible en: https://slowfoodischiaeprocida.wordpress.com [Consulta: 29/07/2020]. Las voces de los activistas locales están registradas en el video del III Congreso en Italia: http:/ / www.terracedlandscapes2016. it/ischia-e-costiera-amalfitana-il-video [Consulta: 29/07/2020]. 
de terrazas en armonía con la naturaleza. Ellas consideran que viven con los principios de la economía circular. Utilizan los recursos locales, como el agua, la tierra, piedras, madera recreando un moderno paisaje local. Reciclan los residuos en compost para la huerta que es la fuente principal de verduras y hierbas con los que se preparan deliciosos potajes para los huéspedes. También producen sus propias semillas. Toda la decoración y los muebles son artesanales, hechos con hojas de palma, lana de oveja. Evitan los subsidios, créditos y prefieren alimentar los lazos de ayuda mutua con los vecinos, amigos y visitantes. Se sienten unidas por valores como el amor por la naturaleza y el cuidado de la salud personal y del ambiente. Los huéspedes que llegan de la ciudad al hostal disfrutan de la vida en el campo, se convierten en un círculo de amigos que regresan con regularidad. Aportan solidariamente con su trabajo en la producción de la huerta y disfrutan de comer sanos y deliciosos productos del campo. ${ }^{27}$

\section{CONCLUSIONES}

Arribamos a un primer plano de conclusiones a partir de la lectura de las representaciones. Estas nos llevan a reconocer que la aplicación de la Investigación Acción Participativa en diferentes momentos de la realización de los cuatro Congresos Mundiales de Terrazas, durante los últimos diez años, han creado oportunidades de intercambio de saberes y mutuo aprendizaje entre campesinos, pobladores y académicos, investigadores, técnicos y activistas de Asia, América Latina, África y Europa. La presencia de los saberes locales en estos eventos ha tenido un impacto empoderador, tanto para los sabios y sabias cuyas experiencias locales continúan ampliándose en espirales autónomas con nuevos impulsos de ideas y acciones así como para otros actores sociales que se han acercado a las percepciones locales enriqueciendo sus criterios de sostenibilidad de los paisajes de terrazas.

Es importante señalar que las representaciones gráficas expresan y transmiten una conciencia y valoración de los sistemas de las terrazas que muestran los siguientes elementos conceptuales:

- La complejidad y diversidad de ideas que sustentan el manejo del espacio y el tiempo de los paisajes imbricados en cosmovisiones y prácticas locales. Cada terraza, cada sistema de terrazas, cada paisaje local tiene características propias optimizando la inclinación de la montaña, la exposición al sol, la variabilidad del clima, el acceso a las fuentes de agua, a la calidad y características del suelo, a la crianza de la biodiversidad posible. La combinación del conjunto de estos factores en la práctica constituyen respuestas culturales originales, y saberes locales, cuya universalidad consiste en que cada cultura ha perfeccionado un saber integral de la vida en las terrazas a partir de una gama de diversas formas de relación íntima con la naturaleza.

- La fineza de las percepciones locales de los cambios en el entramado dinámico de los fenómenos ambientales como el cambio climático, las transformaciones sociales, el abandono de las terrazas o las amenazas como el extractivismo minero, avivan múltiples respuestas locales, como la renovación de

27 Sella y Silvia en el Taller de preparación en Gran Canaria del Iv Congreso Mundial de Terrazas, 2018. Véase también más información sobre la filosofía del hospedaje: https:/ / ecotararetreat.com/es/ inicio [Consulta: 29/07/2020]. 
los calendarios agrícolas, y otras estrategias originales que no sólo se respaldan en los saberes tradicionales, sino que buscan inspiración en otras fuentes de conocimiento.

- La continua recreación y valoración del conocimiento diferenciados por género y generaciones mantienen e incrementan la agrobiodiversidad, articulando la herencia de las semillas de plantas domesticadas y exógenas con características propias de cada valle o región. La transmisión de estos conocimientos confirma la vocación de crianza de la naturaleza en los sistemas de terrazas por las mujeres.

- Los múltiples procesos de búsqueda de diferentes caminos de innovación y creatividad de los saberes agrícolas locales en sintonía con otros sistemas de saberes priorizan la producción de alimentos de acuerdo con las preferencias culturales que contribuyen a la riqueza de las gastronomías locales. Esto nos lleva a plantear que la característica esencial de las terrazas es de carácter agroalimentario.

- La versatilidad de las visiones de futuro de sabios y sabias incluye inspirarse en el pasado, en la recreación de sentimientos de identidad con la tierra, con las plantas, los cultivos y la formación de redes sociales alternativas más allá del trabajo familiar y de repensar el bienestar colectivo, en lugar del simple crecimiento económico.

Un segundo plano de conclusiones son las propuestas de acción que surgieron en la sesión final del Iv Congreso Mundial de Terrazas «Re-encantar bancales» en La Gomera en marzo 2019. Los congresistas elaboraron visiones de futuro sobre los cuatro ejes temáticos: Habitar, Aprender, Saber y Construir (ITLA: 2020).

Resumimos el producto de las reflexiones sobre la sostenibilidad de los saberes de los paisajes de terrazas en las siguientes propuestas de acción (Figura 21):

I) Diversificar la producción de alimentos orgánicos en las terrazas en pequeña escala y que los productores logren condiciones de vida saludables y de Buen Vivir.

II) Crear redes de conocimiento en un esfuerzo colectivo entre las generaciones con una base ética de respeto a la naturaleza que regenere las comunidades rurales.

III) Vivir con dignidad, con alternativa innovadora de Buen Vivir en el campo, sin copiar modelos urbanos.

IV) Crear laboratorios de conocimiento que sean espacios de reflexión y de experimentación con diferentes tradiciones de conocimiento respetando la diversidad y las visiones de futuro.

v) Generar inventarios de soluciones prácticas tradicionales y con una perspectiva agroecológica que beneficie a la naturaleza y a los seres humanos.

vi) Promover Diálogos de Saberes entre diferentes sistemas de conocimiento, vinculando a la generación de los mayores con los científicos con la finalidad de re-encantar la vida en los paisajes de terrazas.

VII) Establecer canales de comunicación entre todos los actores para que sean capaces de implementar acciones desde abajo sin obstáculos burocráticos (ITLA, 2020).

Finalmente concluimos en un plano reflexivo, la mayor parte del mundo está alimentado por la pequeña agricultura, y las terrazas como parte de ese variado mosaico de sistemas de saberes bioculturales invita a mirarlos desde otros prismas de poder y con nuevos valores que amplíen nuestros horizontes intelectuales. La diversidad de saberes bioculturales contiene una gran riqueza epistemológica y su poder transformador se evidencia en la gama plural de 
múltiples vías de pensar, sentir, actuar y de vivir en paisajes moldeados con creatividad cautivadora. Las nuevas tecnologías de digitalización y el poder corporativo de la agroindustria se legitiman descartando los aportes de los saberes de otras tradiciones epistemológicas causando el epistemicidio, o sea las diferentes formas de exclusión de los saberes colectivos implantando un solo sistema de conocimiento atribuyéndose ostentar superioridad epistemológica. Así se elimina un valioso elemento para la sostenibilidad de la vida en el planeta (SANTOS, 2014).

El enfoque de la diversidad biocultural de los saberes pone en relieve la relación de mutua influencia, de crianza como una inextricable reciprocidad con la naturaleza. Es decir, los agricultores transforman el paisaje con sus concepciones culturales, y a su vez la naturaleza y el paisaje agrícola transformado impactan en la experiencia particular de un grupo, tiñendo sus identidades y visiones, modos de pensar, vivir, producir y comer. Este es un aporte conceptual para tomar en cuenta en el debate sobre la sostenibilidad.

Reconocer las contribuciones epistemológicas que orientan los estilos de vida de quienes producen alimentos de calidad en paisajes de terrazas practicando agriculturas que no destruyen el medio ambiente, ni la salud de la gente, permite repensar alternativas para sociedades con justicia cognitiva. Ésta es posible creando espacios democráticos de diálogo de saberes entre los/las custodios/as de terrazas, investigadores, activistas, desplegando la imaginación, recordando la memoria de los paisajes de terrazas y de formas de vida plena sin exclusión de las más distintas tradiciones epistemológicas. Por ello, la sostenibilidad no puede eludir como horizonte moral el apoyo a la continuidad y la recreación de muchas maneras de pensar, vivir la vida y de producir con los saberes transformadores de los paisajes de terrazas.

\section{AGRADECIMIENTOS}

Queremos expresar nuestro agradecimiento y homenaje a campesinos y campesinas, técnicos y técnicas, estudiantes, profesionales, activistas quienes participaron en la elaboración de los testimonios gráficos y contribuyeron a las reflexiones sobre los saberes en paisajes de terrazas.

\section{REFERENCIAS}

Acosta, A. (2010): El Buen Vivir en el camino del post-desarrollo - Una lectura desde la Constitución de Montecristi, Friedrich Ebert Stiftung - ILDIS, Quito.

Alberti, F. et alii, eds. (2018): Terraced Landscapes: choosing the future, Regione del Veneto, Venezia.

Baguilat, C. (2012): "Conservation and development of the rice terraces of the Philippine Cordilleras as a world heritage site», en H. Peters y J. SHI (eds.), First Terraced Landscapes Conference (Honghe - China), Yunnan People's Publishing House, Kunming.

BERMAN, M. (1981): The reenchantment of the world, Cornell University Press, Cornell. Bouchery, P. (2010): «Irrigation System and Religious Interpretation of the Local

Environment among the Hanis in Yunnan», en M. LeComte-Tilouine, Nature, 
Culture and Religion at the Crossroads of Asia, Social Science Press, Routledge, Abingdon: 318-342.

BROKHENSHA, D.; WARren, M.; Werner, O., eds. (1980): Indigenous Knowledge Systems and Development, University of America Press, Lanham

Childe, G. (1956): Society and knowledge, George Allan \& Unwin Ltd., London.

ConkLin, H. (1980): Ethnographic Atlas of Ifugao: A Study of Environment, Culture, and Society in Northern Luzon, American Geographical Society of New York, New York.

CooK, N.D. (2011): Los hijos del volcán, dualidad andina en el Valle del Colca, Ediciones El Lector, Lima.

CoWAN, C.W.; WATSON, P.J., eds. (1992): The origins of agriculture, an international perspective, Smithsonian Institution Press, Washington.

DonkIN, R.A. (1979): Agricultural terracing in the Aboriginal New World, Viking Fund Publications in Anthropology, Tucson, Arizona.

EARLS, J. (2015): «Inka cosmology in Moray: Astronomy, agriculture and pilgrimage», en I. SHIMADA, The Inka empire, a multidisciplinary approach, University of Texas Press, Austin: 121-147.

Eco, U.; Zorzoli, G.B. (1962): Historia ilustrada de los inventos, Fabril, Buenos Aires. EINARSEN, J., ed. (1995): The sacred mountains of Asia, Shambala, Boston.

ETC-Group (2017): ¿Quién nos alimentará? La red campesina alimentaria o la cadena agroindustrial, ETC, Val David, Canadá.

FALs BORDA, O. (1984): El problema de como investigar la realidad para transformarla en la praxis, Tercer mundo editores, Bogotá.

FALS BORDA, O. (1992): «La ciencia y el pueblo: nuevas reflexiones», en M. SALAZAR, La investigación-acción participativa. Inicios y desarrollos, Cooperativa Editorial Magisterio, Bogotá: 65-84.

FAO (2018): Sistemas importantes del patrimonio agrícola mundial. Globally Important Agricultural Heritage Systems (GIAHS). Combining agricultural biodiversity, resilient ecosystems, traditional farming practices and cultural identity, FAO, Roma.

Fuller, D.; WeIsSKopf, A.; CовO, C. (2016): «Pathways of rice diversification across Asia», Archaeology international, 19: 84-96.

GavazzI, A. (2010): Arquitectura Andina. Formas e historia de los espacios sagrados, Apu Graph Editores, Lima.

GuDYNAS, E. (2011): Buen vivir: Germinando alternativas al desarrollo, ALAI, Quito.

Guimbatan, R.; Baguilat, T. (2006): «Misunderstanding the notion of conservation in the Philippine rice terraces-cultural landscapes», International Social Science Journal, 58 (187): 59-67. https:/ / doi.org/10.1111/j.1468-2451.2006.00606.x.

Havelock, R. (1986): «The knowledge perspective: Definition and scope of a new study domain», en G. Beal, W. Dissayanake y S. Konoshima, Knowledge generation, exchange and utilization, Westview, Boulder: 11-34.

ITLA (2020): Conclusiones IV Congreso ITLA de La Gomera, Islas Canarias. ITLA, San Sebastián, La Gomera.

Kieninger, P.R.; YamajI, E.; PenKer, M. (2011): «Urban people as paddy farmers: The Japanese Tanada Ownership System discussed from a European perspective», Renewable Agriculture and Food Systems: 26 (4): 328-341.

Kimbrell, A., ed. (2002): Fatal harvest-the tragedy of industrial agricultura, Island Press, Washington.

LAIRD, S., ed. (2002): Biodiversity and traditional knowledge. Equitable partnerships in 
El poder transformador de los saberes en paisajes de terrazas

practice, Earthscan, London.

LÉvi-Strauss, C. (1964): El pensamiento salvaje, Fondo de Cultura Económica, México.

Marglin, S. (2000): Perdiendo el contacto. Hacia la descolonización de la economía, Pratec, Lima.

Marglin, S. (2006): Dominating knowledge. Development, culture and resistance, Clarendon Press, Oxford.

MAFFI, L., ed. (2001): On biocultural diversity-linking language, knowledge and the environment, Smithsonian Institution Press, Washington.

MoOney, P. (2019): La insostenible agricultura 4.0-Digitalización y poder corporativo en la cadena alimentaria, Grupo ETC, México.

Pérez SÁnchez, J.M. (2014): Agricultura de terrazas en Tlaxcala: La Caridad Cuaxonacayo, Gobierno Tlaxcala, México.

Peters, H., ShI, J. (2012): First Terraced Landscapes Conference. Paper collection, Yunnan Publishing House, Kunming.

Phuntsho, K. (2013): The history of Bhutan, Random House India, Gurgaon.

PIMBERT, M., ed. (2018): Food Sovereignty, agroecology and biocultural diversity. Constructing and contesting knowledge, Routledge, Earthscan, Oxon.

POSEY, D., ed. (1999): Cultural and spiritual values of biodiversity, UNEP, Nairobi.

Prain, G.; FujISAKa, S.; WARren, M.D. (1999): Biological and cultural diversity. The role of indigenous agricultural experimentation in development, IT Publications, London.

SALAS, M. (2013): Los sabores y las voces de la tierra. Visualizando la soberanía alimentaria en los Andes, IIED-PASA, London.

Salas, M.; Tillmann, T. (2010): Participatory Action Research: Embracing the knowledge perspective within field research, RCSD, Chiang Mai.

SANDOR, J.A. (2006): «Ancient Agricultural Terraces and Soils», en B. P. WARKENTIN, Footprints in the Soil. People and Ideas in Soil History, Elsevier, Amsterdam: 505534.

SANTOS, B.S., coord. (2004): Democratizar la democracia. Los caminos de la democracia participativa, Fondo de Cultura Económica, México

SANTOS, B.S. (2014): Epistemologies of the South, justice against epistemicide, Paradigm, Boulder.

SANTOSOMBAT, Y. (2003): Biodiversity, local knowledge and sustainable development, RCSD, Chiang Mai.

Sмiтн, B.D. (1994): The emergence of agricultura, Scientific American Library, New York.

Toledo, V.M., BARRERA-BASSOLS, N. (2008): La memoria biocultural. La importancia de las sabidurías tradicionales, Icaria, Barcelona.

Tillmann, T. (1997): Las estrellas no mienten. Agricultura y ecología subjetiva andina en Jauja, Peru, Abya-Yala, Quito.

Tillmann, T.; Bueno de Mesquita, M. (2014): Encuentro de culturas y saberes de terrazas del mundo. II Congreso Internacional de Terrazas, CBC - JICA, Cusco.

Tillmann, T.; VARotTo, M. (2016): «9 - Heritage and historical rural landscapes - Ischia e Costiera Amalfitana», en F. Alberti, A. Dal Pozzo, D. Murtas, M. A. Salas y T. Tillmann, Paesaggi terrazzati: Scelte per il Futuro, Regione del Veneto, Venezia: 107-114.

UNESCO (2008): Sustainable tourism and the preservation of the world heritage site of the Ifugao Rice Terraces, Philippines, IMPACT, Bangkok 
VAROtTo, M.; BonARd, L.; TARolli, P. (eds.) (2019): World terraced landscapes: history, environment, quality of life, Springer, Cham.

VAvilov, N.I. (1992): Origin and Geography of Cultivated Plants, Cambridge University Press, Cambridge.

VisvanATHAN, S. (2006): «Official hegemony and contesting pluralisms», en G. LiNS y A. Escobar (eds.), World Anthropologies. Disciplinary transformations within systems of power, Berg Publishers, Oxford: 239-258.

WATSON, E. (2009): Living terraces in Ethiopia. Konso landscape, culture and development, James Currey, Suffolk.

Weikenmeier, P.; Weikenmeier, A. (1994): Jemen. Landschaft, Menschen, Kulturgeschichte, Belser Verlag, Zürich.

Zuidema, T. (2010): El calendario Inca. Tiempo y espacio en la organización ritual del Cuzco. La idea del pasado, Fondo Editorial del Congreso del Perú, Lima. 


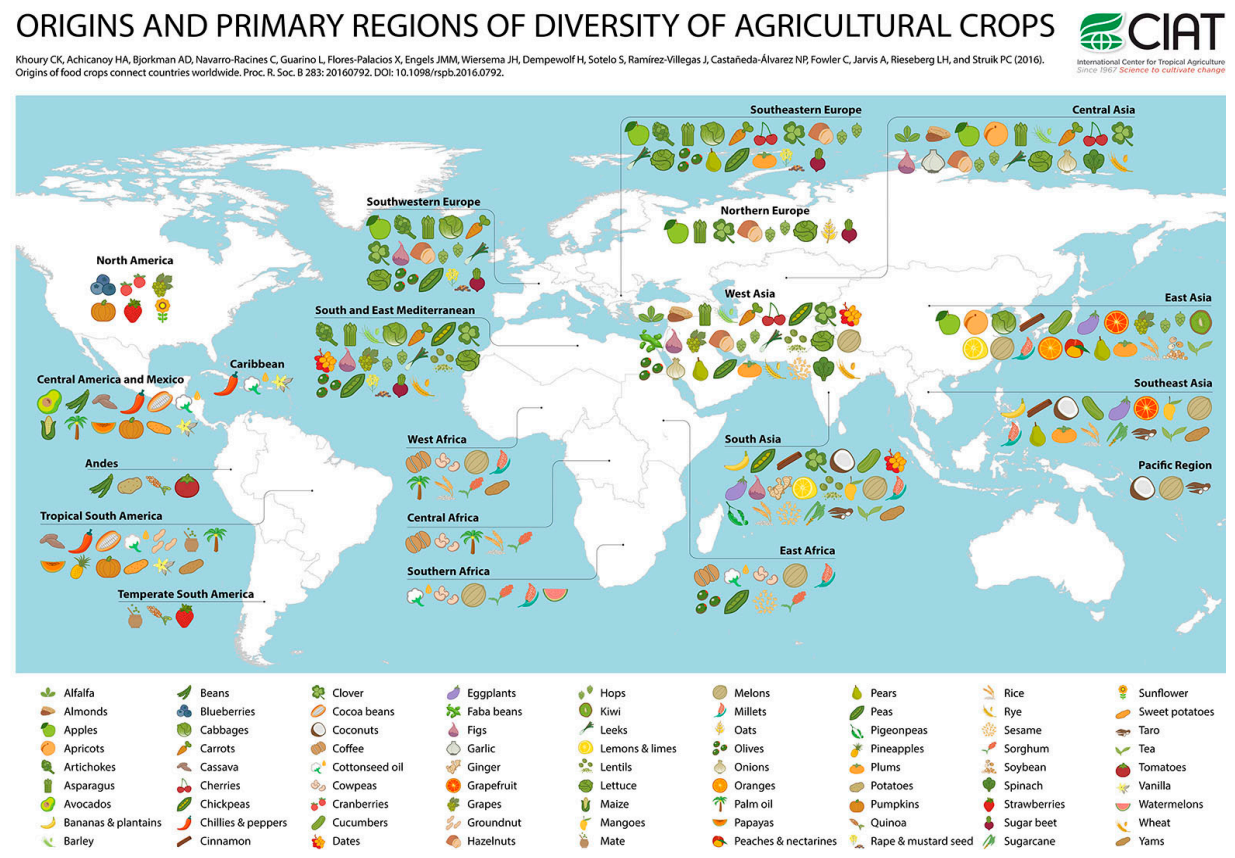

Figura 1. Orígenes de la diversidad los cultivos (KHOURY, 2016).

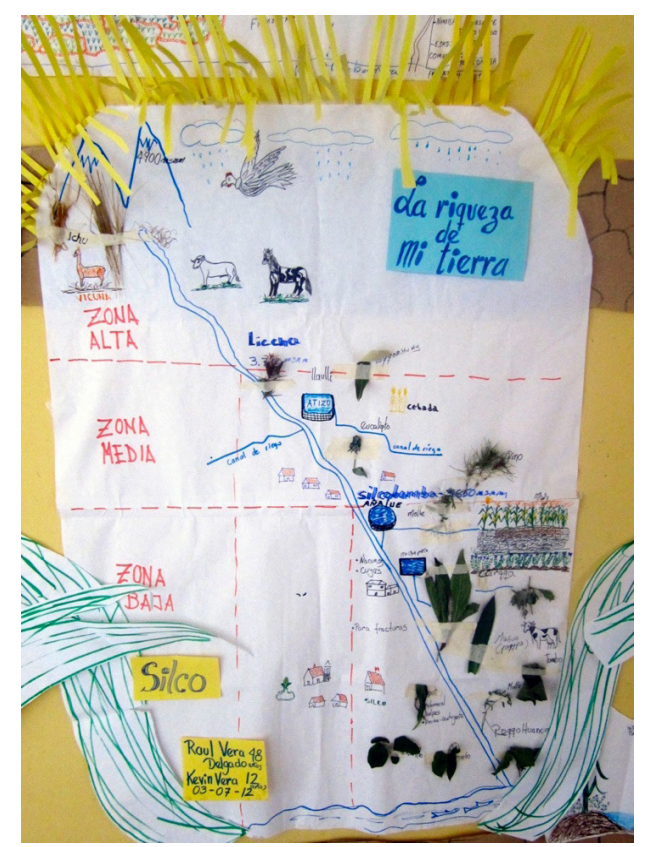

Figura 2. Perfil de las tres zonas agropecuarios de Silco. Fotografía: Tillmann. 


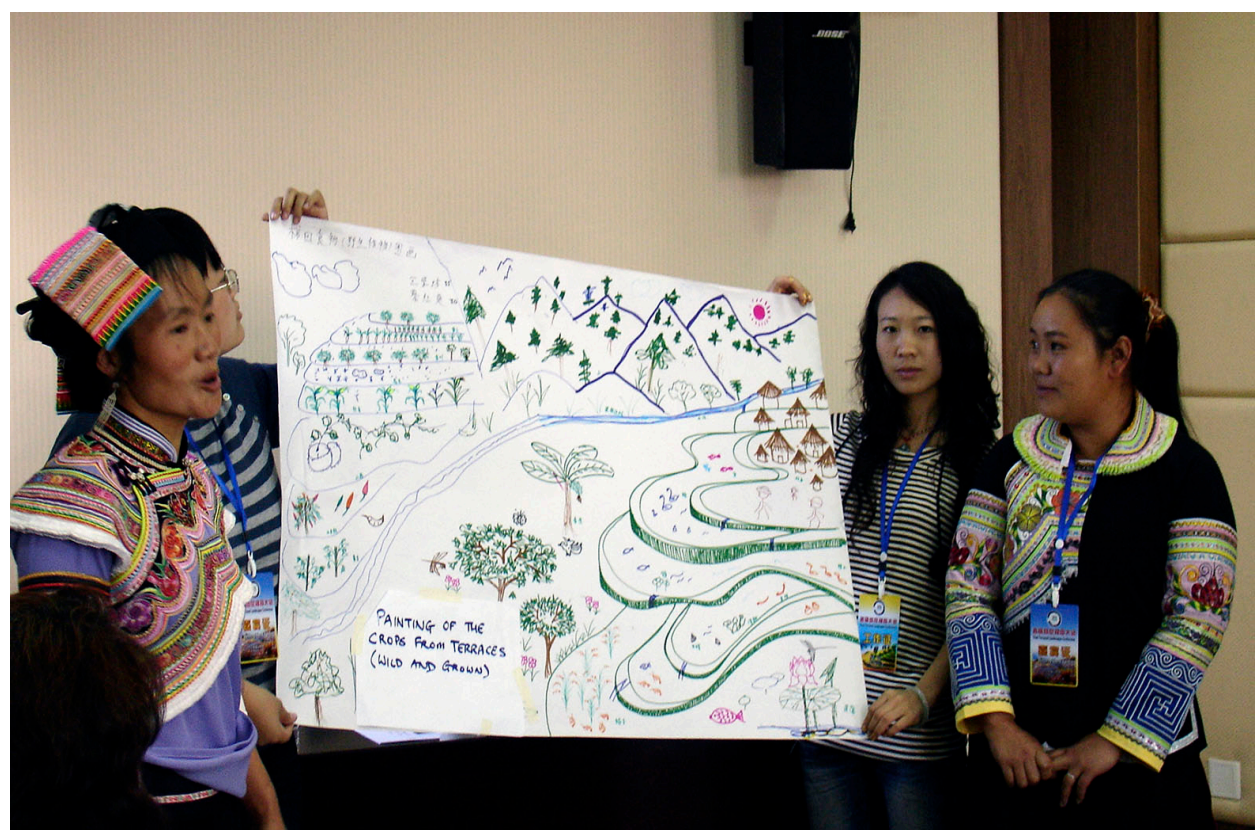

Figura 3. Mapa del sistema de terrazas y las zonas de cultivo de Yuanyang. Fotografía: Tillmann.

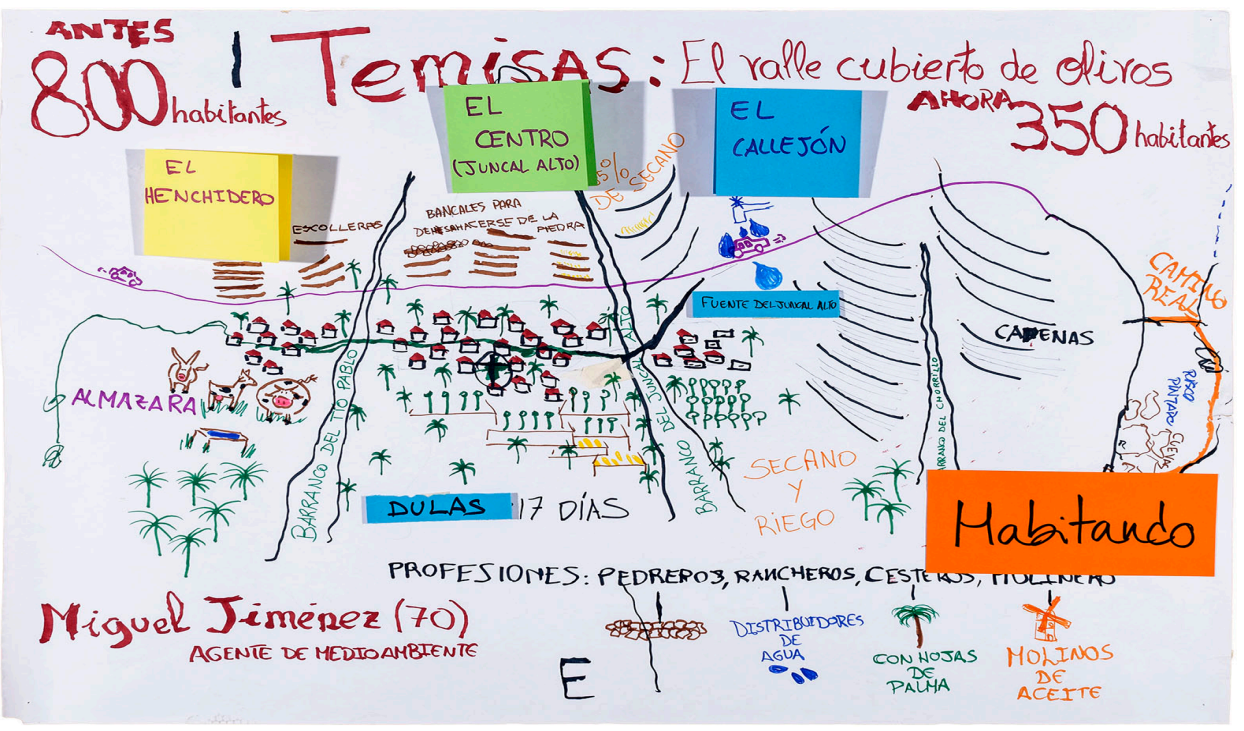

Figura 4. Mapa del pueblo y las terrazas de Temisas. Fotografía: Santana. 


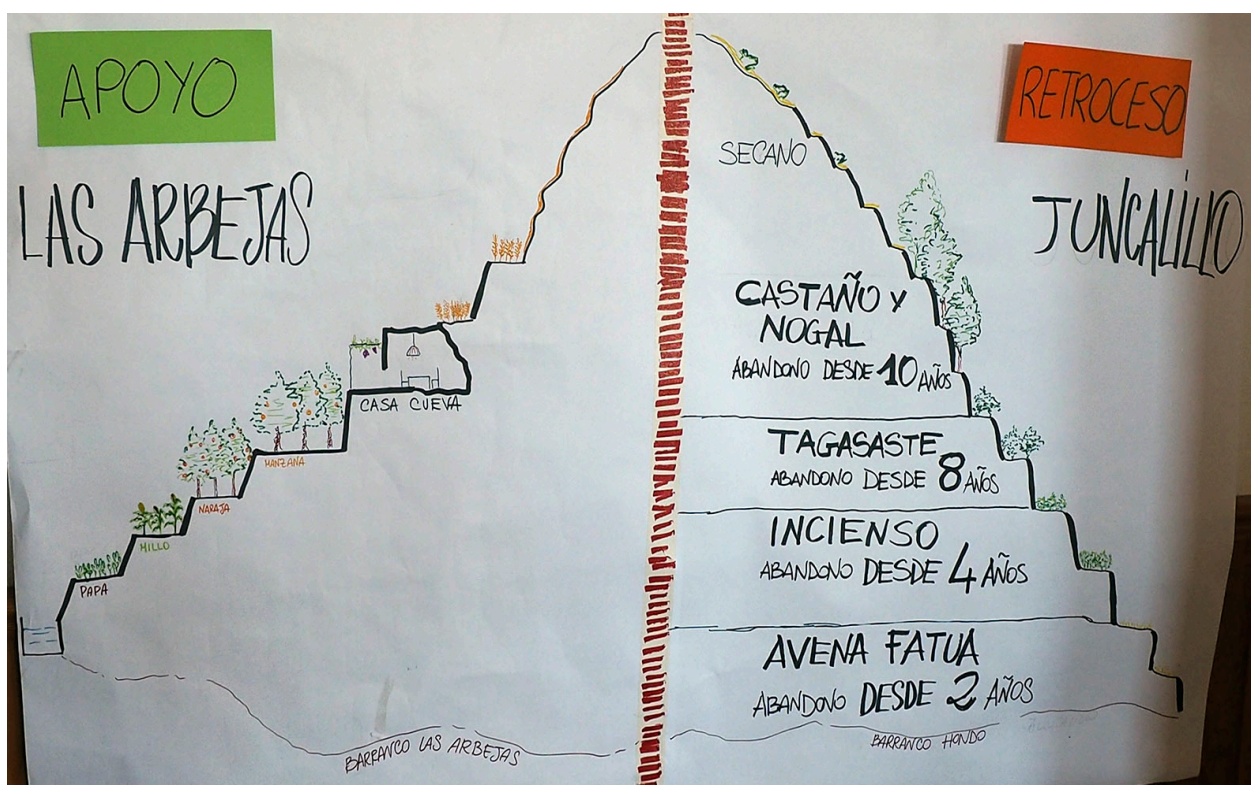

Figura 5. Perfil de dos tipos de laderas con bancales en Barranco Hondo. Fotografía: Tillmann
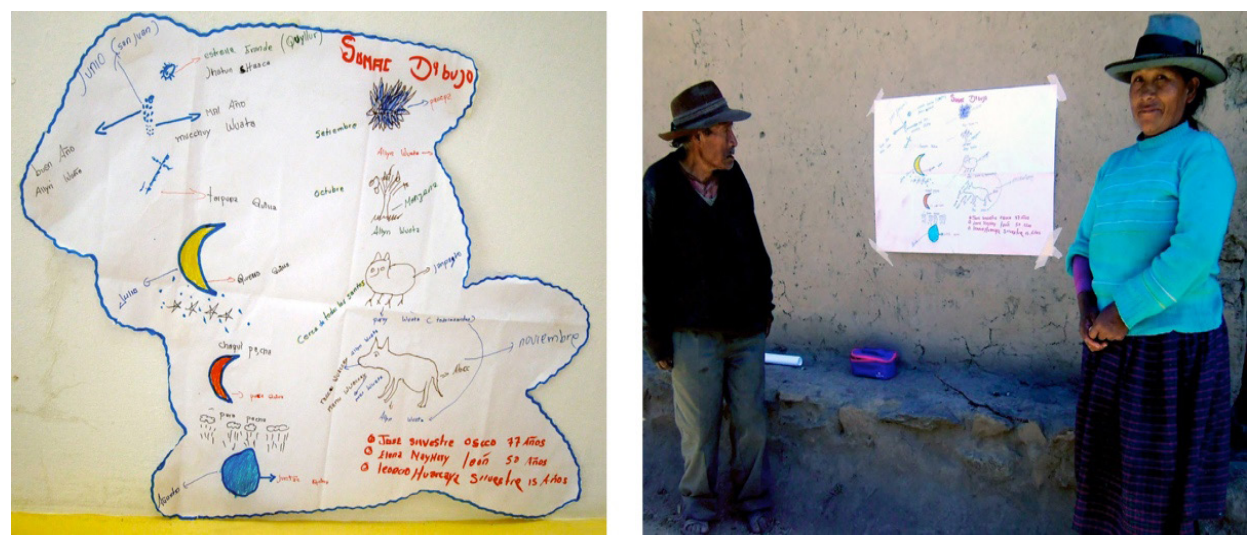

Figuras 6. Calendario agro-astronómico de Mollebamba, Perú. José y Elena presentan su dibujo sobre las estrellas y la luna una vez terminado. Fotografía: Tillmann. 


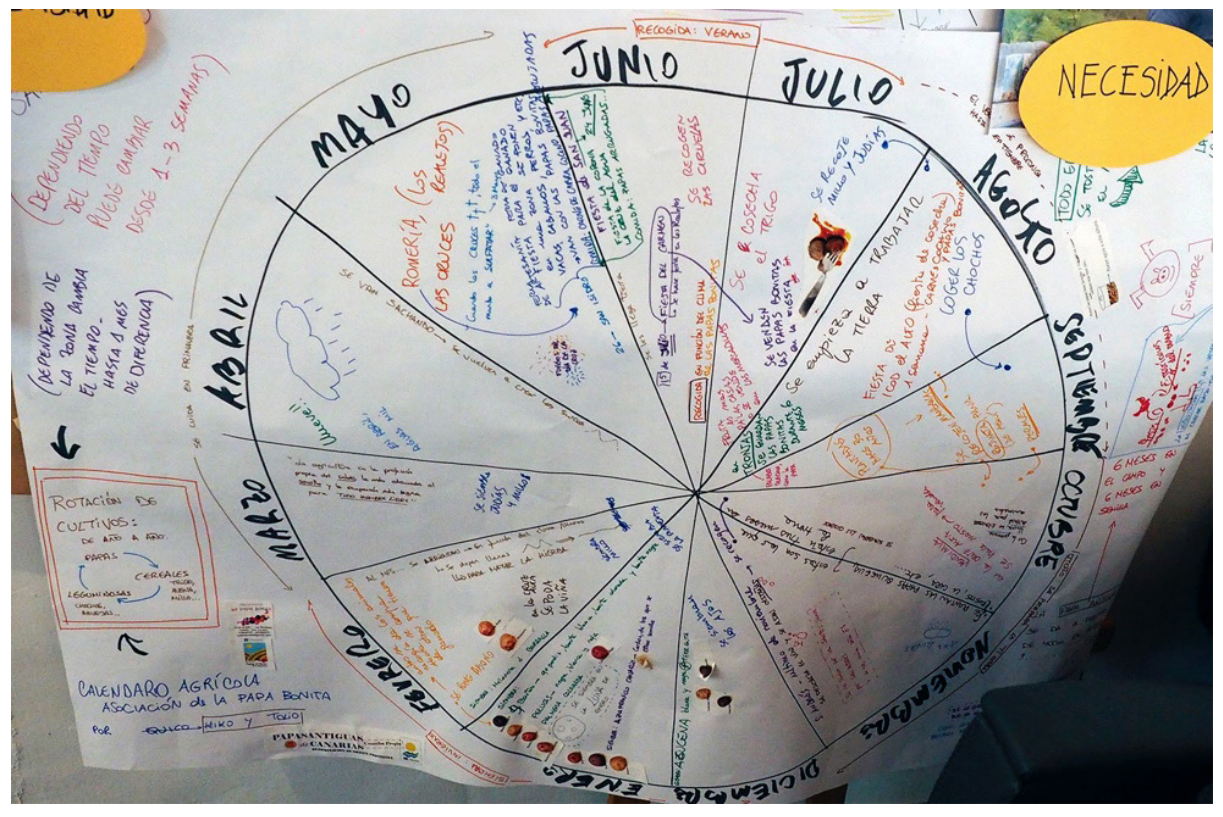

Figura 7. Ciclo agrícola de la papa bonita en Los Realejos de Tenerife. Fotografía: Tillmann. 


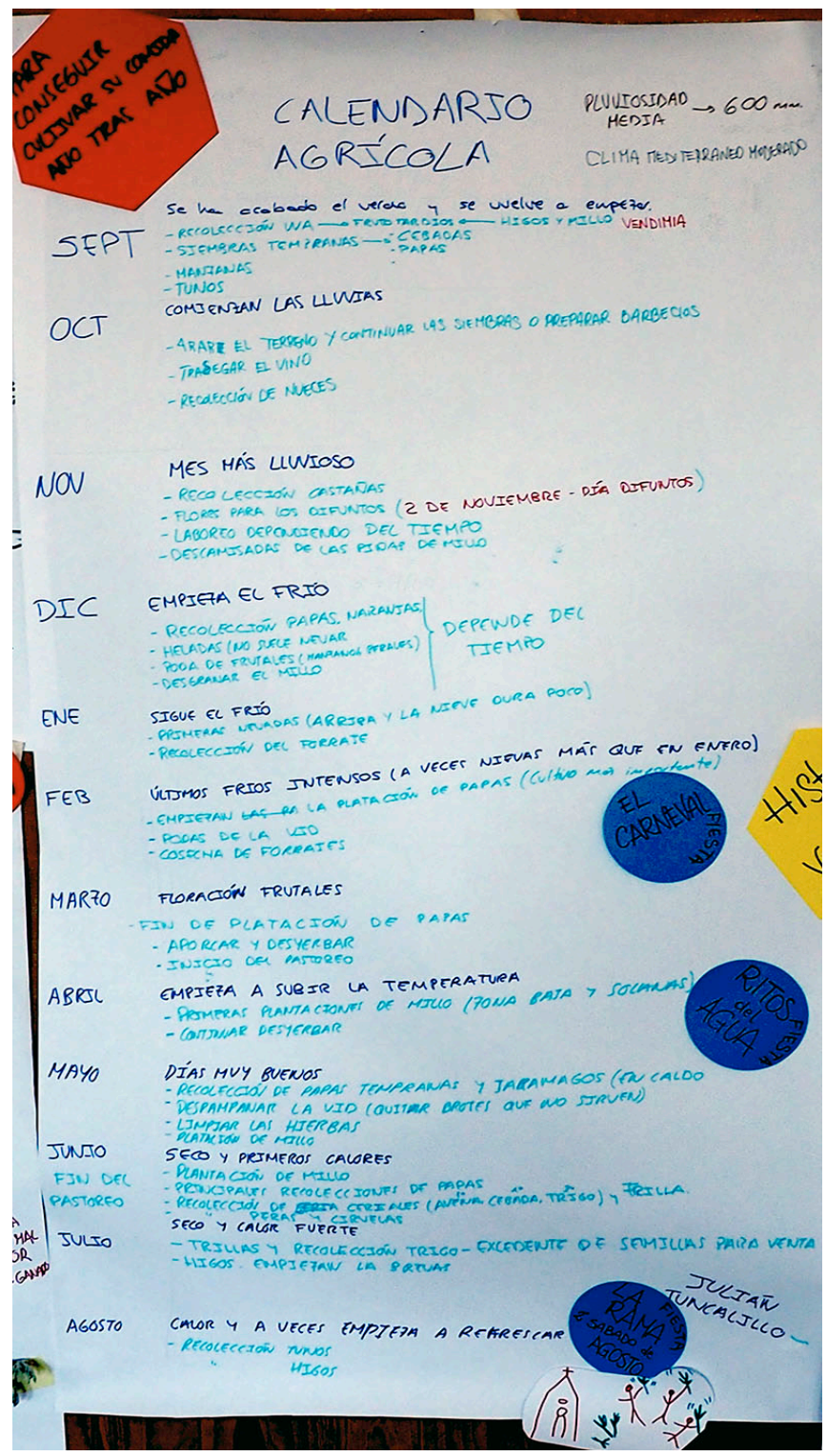

Figura 8. Calendario agrícola (características climáticas mensuales y labores agrícolas). Fotografía: Tillmann. 
María Angélica Salas y Timmi Tillmann

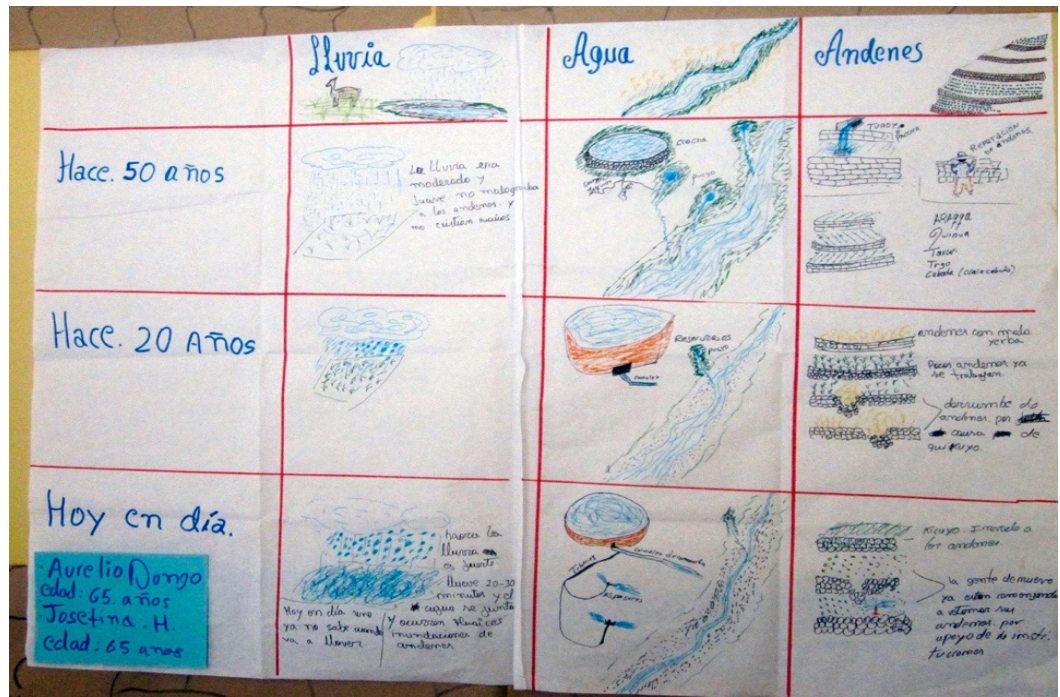

Figura 9. Diagrama histórico de cambio climático. Fotografía: Tillmann.
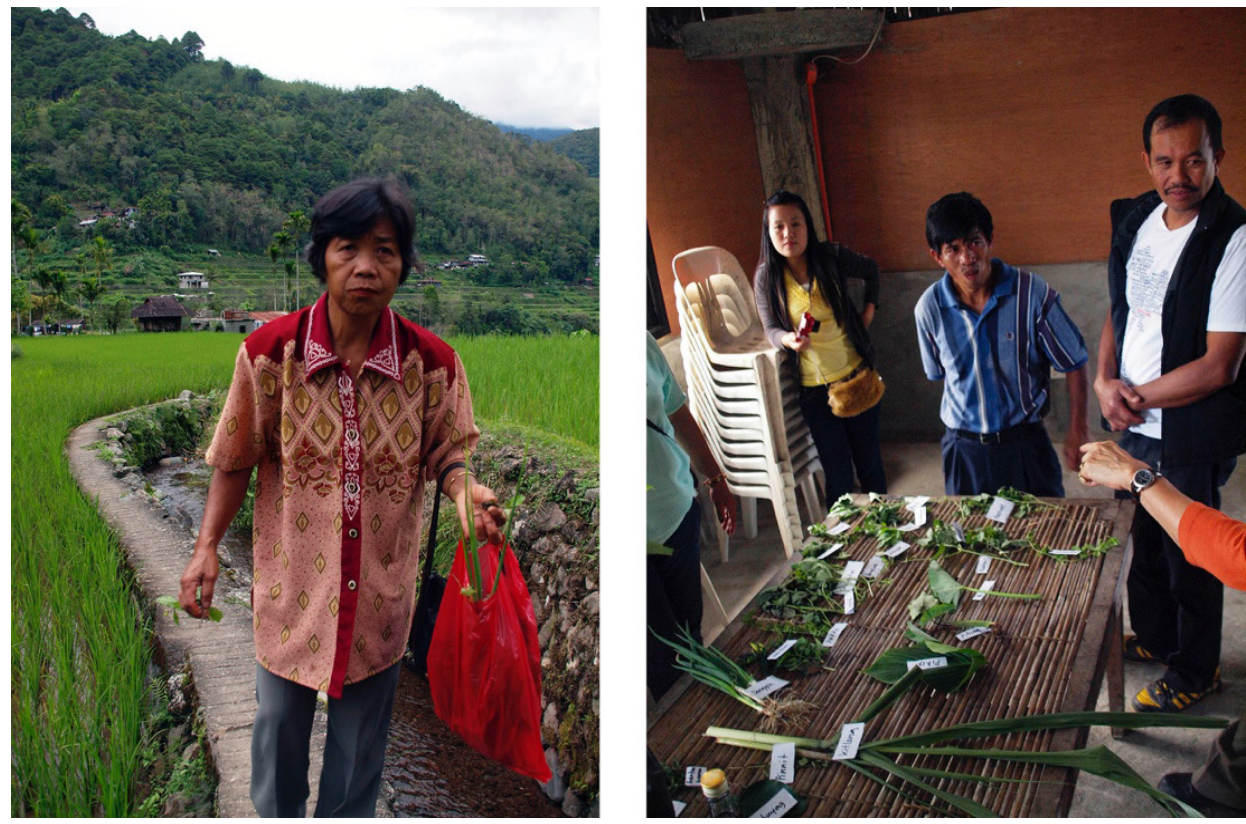

Figura 10. Izquierda: Recorrido para la colección de plantas; derecha: Clasificación de plantas con nombres locales. Fotografías: Tillmann. 


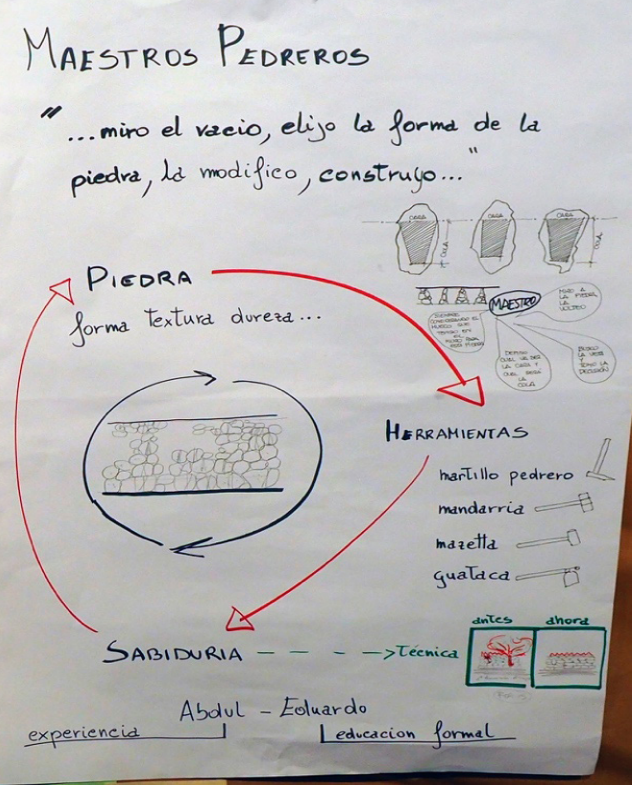

Figura 11. Diagrama de saber del pedrero. Fotografía: Tillmann.

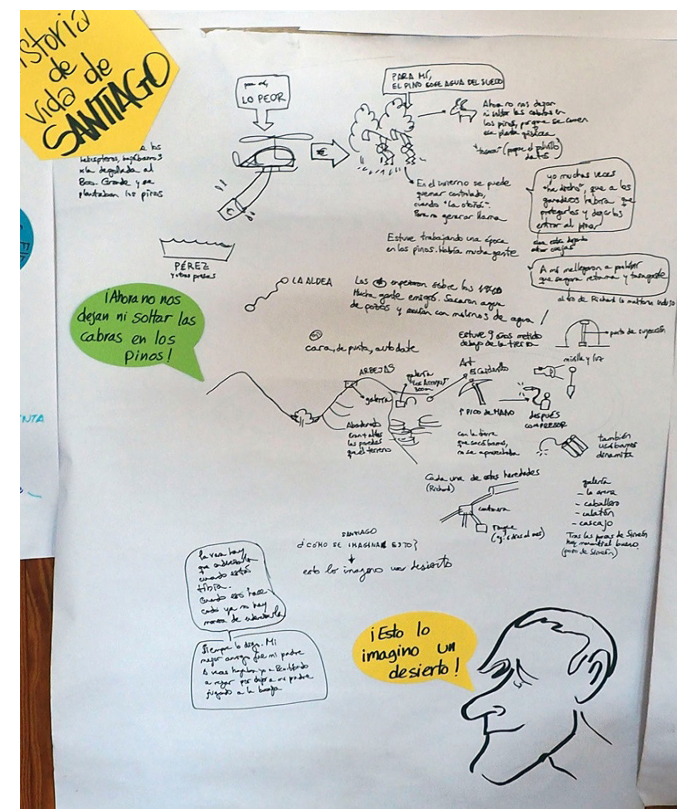

Figura 12. Biografía del sabio de las galerías. Fotografía: Tillmann (Jiménez). 


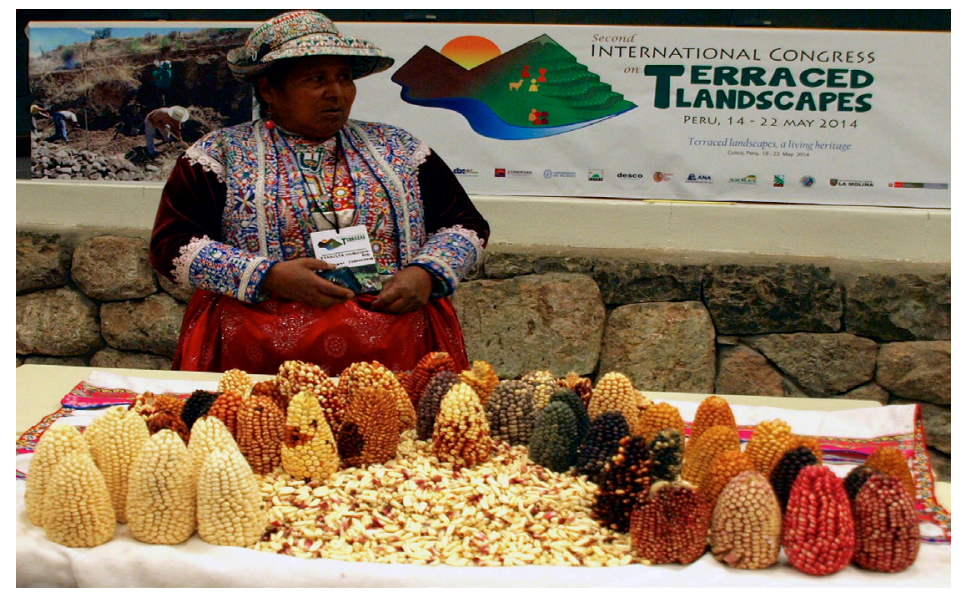

Figura 13. La guardiana del maíz de Cabanaconde. Fotografía: Tillmann.

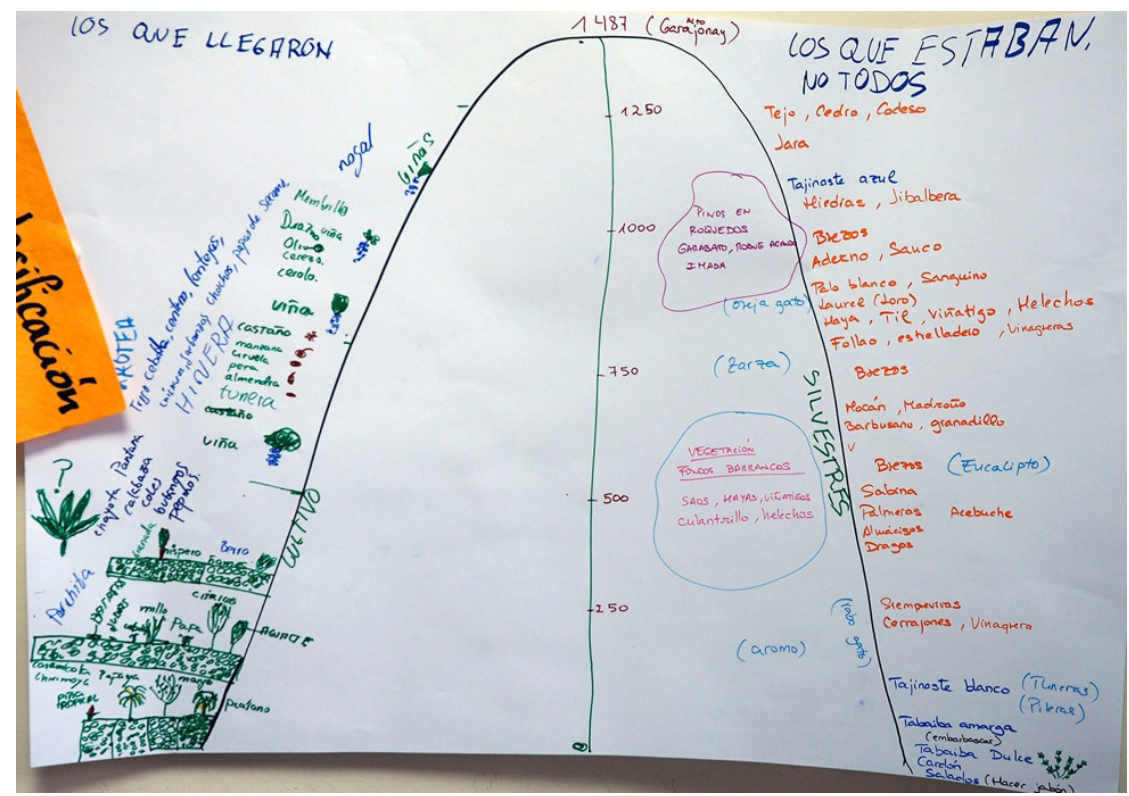

Figura 14. Perfil con la clasificación de plantas exóticas y endémicas. Fotografía: Tillmann. 
El poder transformador de los saberes en paisajes de terrazas

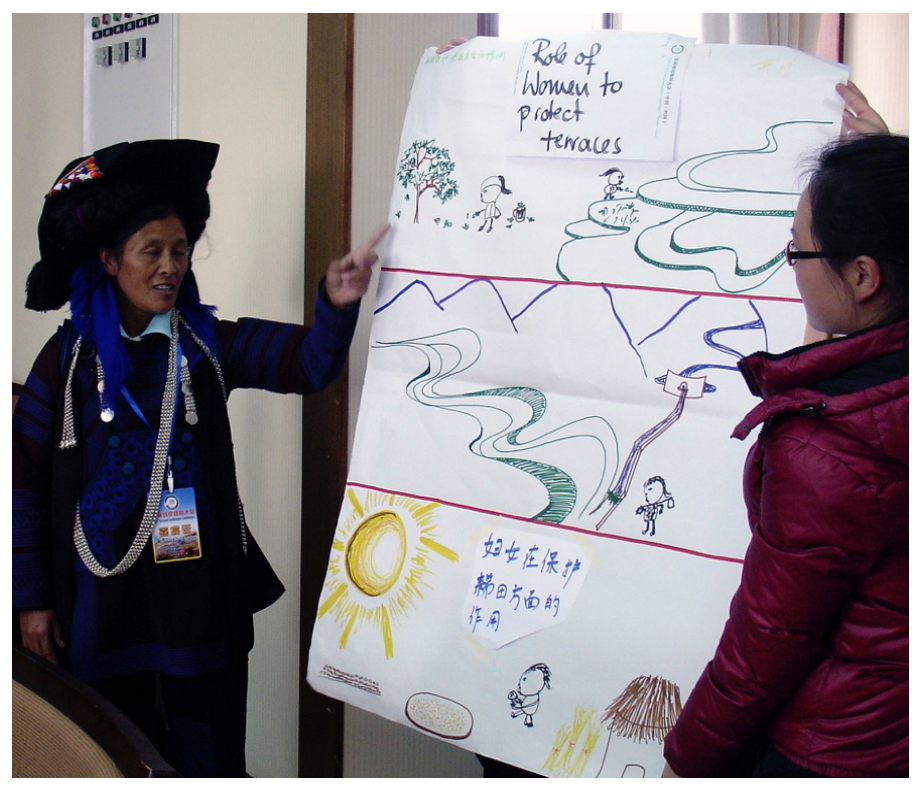

Figura 15. Dibujo de una mujer Hani explicando cómo conserva las semillas y protege las terrazas. Fotografía: Tillmann.

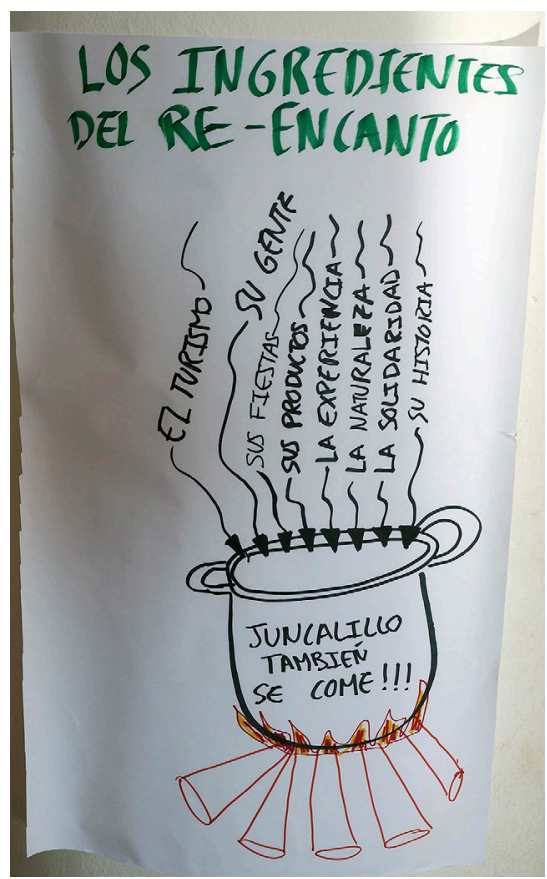

Figura 16. Mapa mental de los ingredientes del reencanto. Fotografía: Tillmann. 


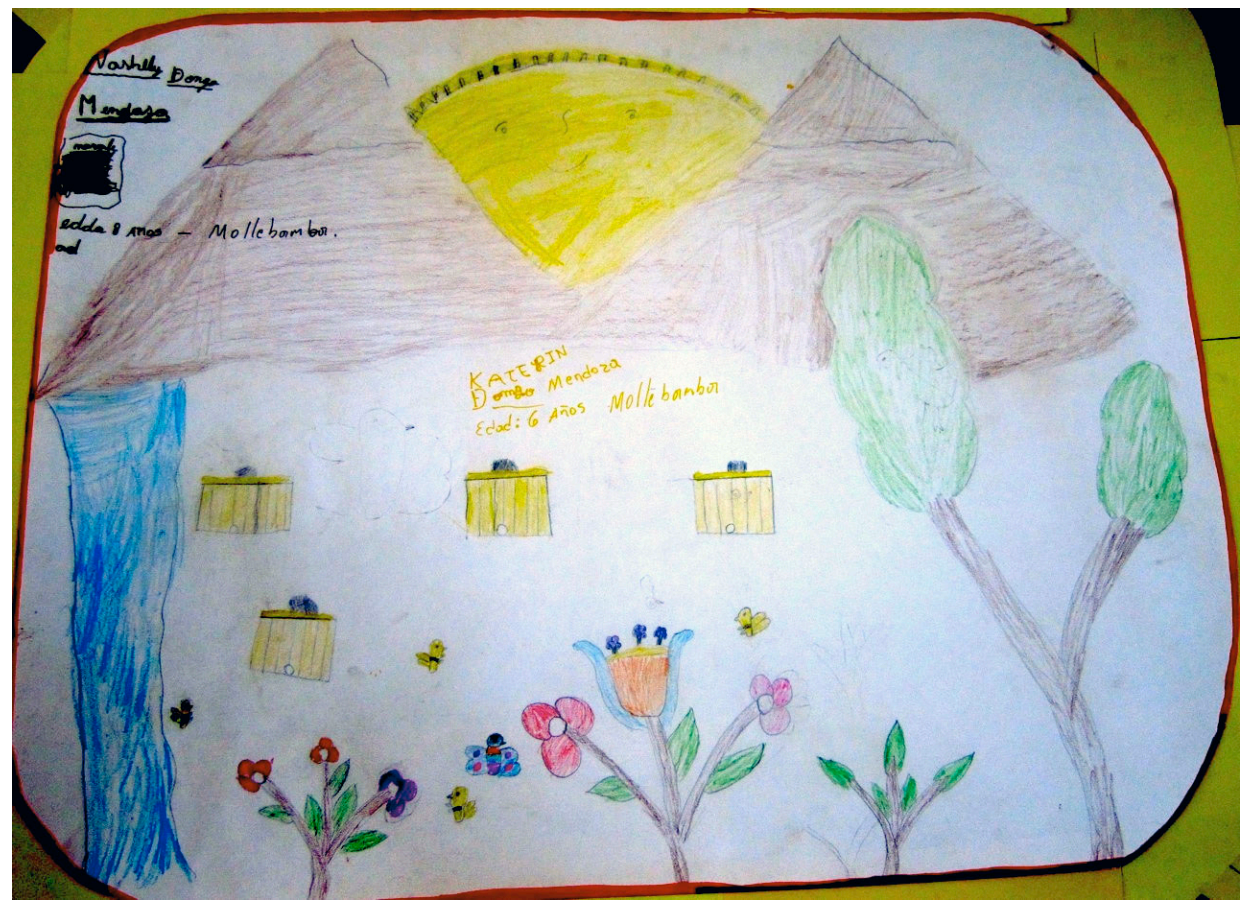

Figura 17. Dibujo de futuro de un grupo de niños andinos. Fotografía: Tillmann.

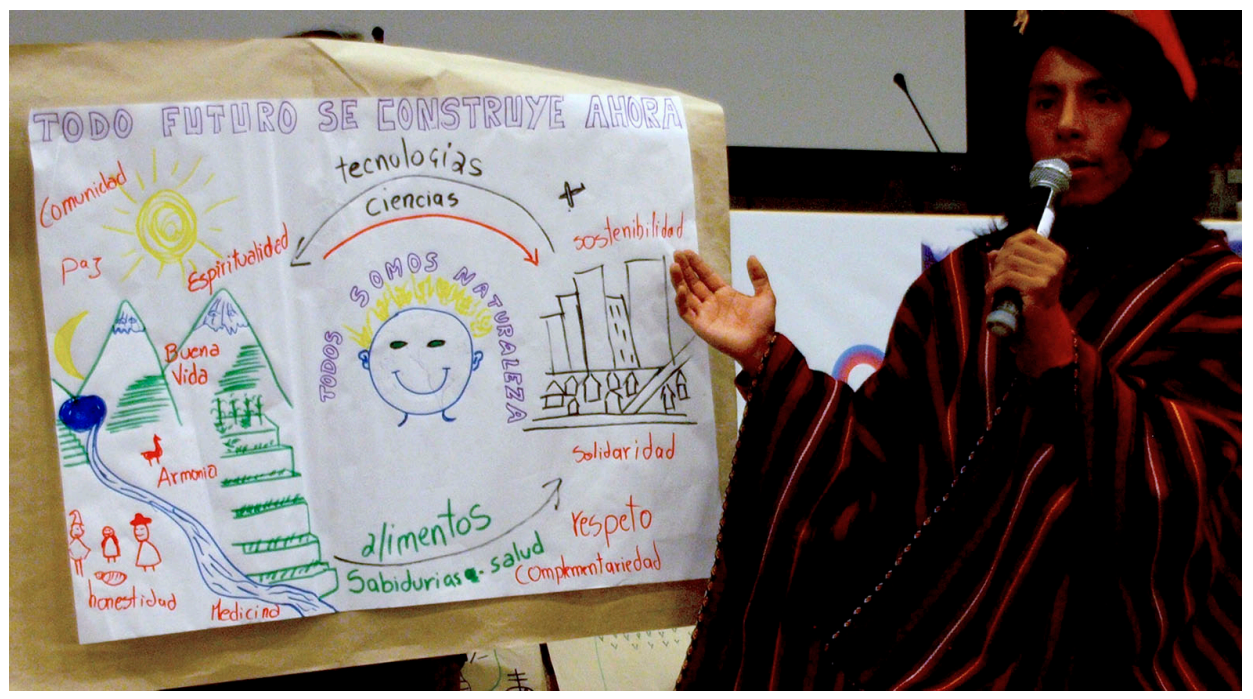

Figura 18. Dibujo del futuro de jóvenes andinos inspirados en Moray, centro de experimentación Inca. Fotografía: Tillmann/Salas. 


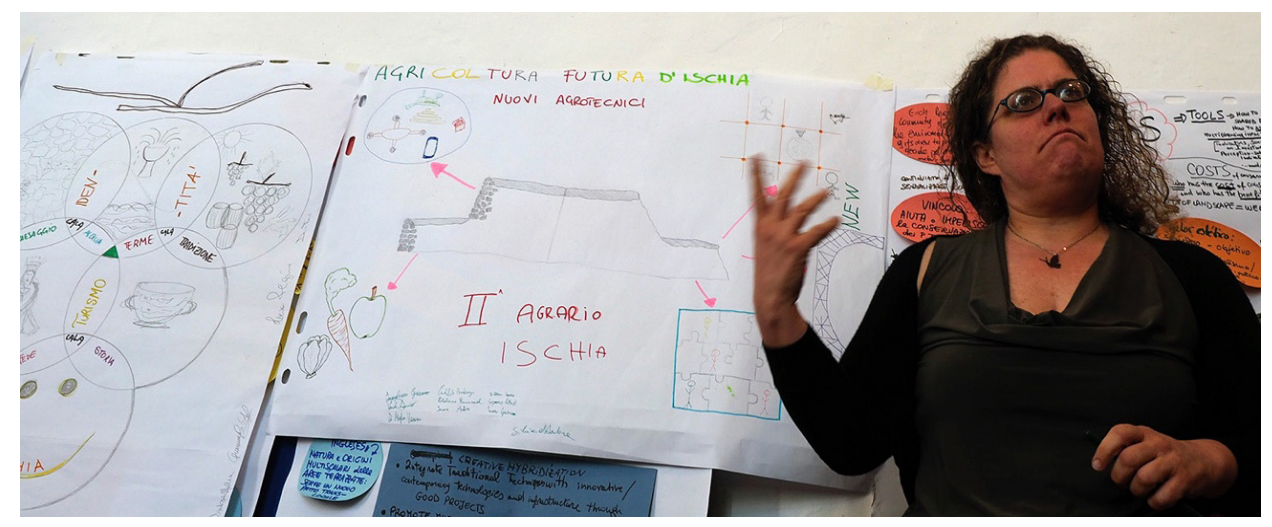

Figura 19. Dibujo de la agricultura futura de Ischia. Fotografía: Tillmann.

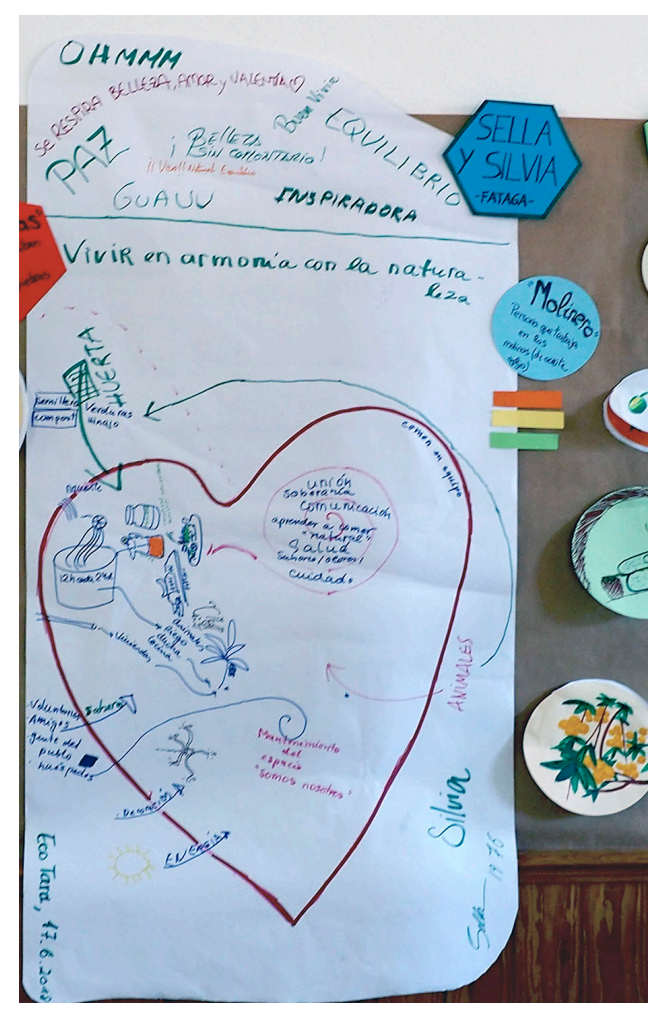

Figura 20. Visión de futuro de las activistas ecológicas de Fataga. Fotografía: Santana. 


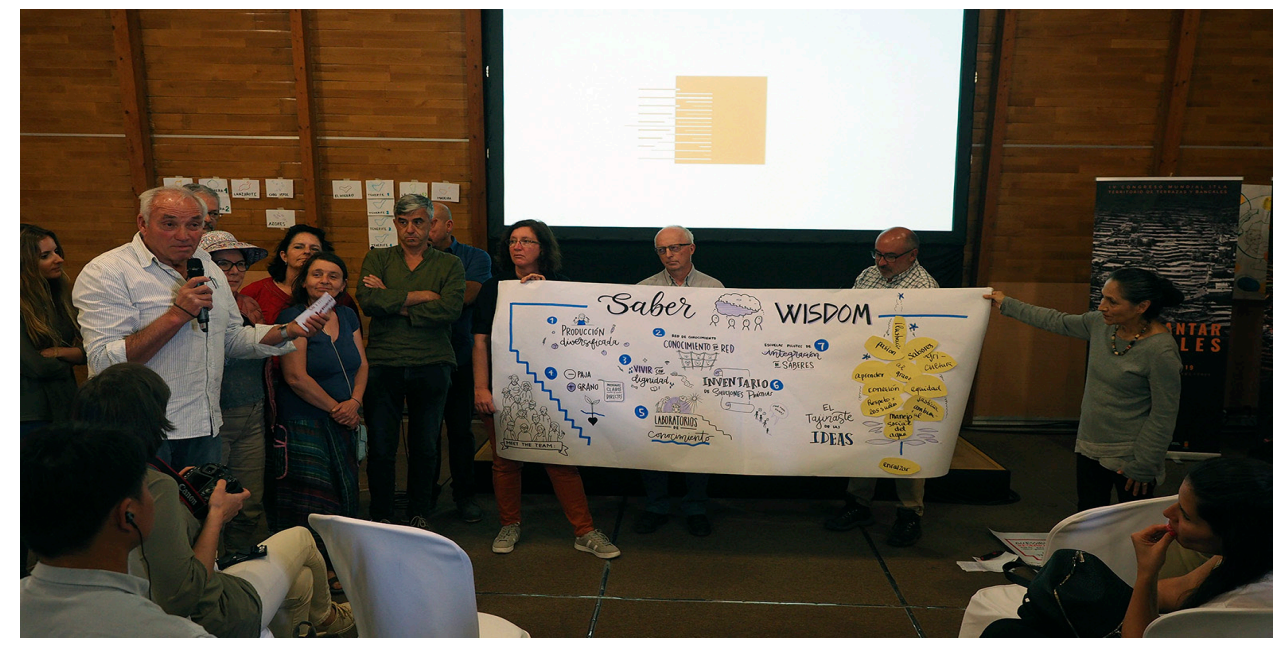

Figura 21. Visualización de las propuestas de acción del tema saber en el Iv Congreso. Fotografía: Tillmann. 
